\title{
Calcium dysregulation mediates mitochondrial and neurite outgrowth abnormalities in SOD2 deficient embryonic cerebral cortical neurons
}

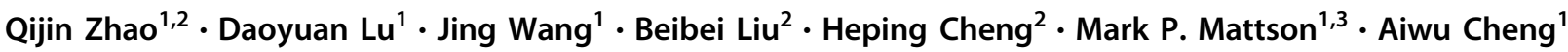

Received: 24 July 2018 / Revised: 17 October 2018 / Accepted: 22 October 2018 / Published online: 2 November 2018

( ) ADMC Associazione Differenziamento e Morte Cellulare 2018

\begin{abstract}
Mitochondrial superoxide dismutase 2 (SOD2) is a major antioxidant defense enzyme. Here we provide evidence that SOD2 plays critical roles in maintaining calcium homeostasis in newly generated embryonic cerebral cortical neurons, which is essential for normal mitochondrial function and subcellular distribution, and neurite outgrowth. Primary cortical neurons in cultures established from embryonic day $15 \mathrm{SOD}^{+/+}$and SOD $2^{-/-}$mice appear similar during the first $24 \mathrm{~h}$ in culture. During the ensuing two days in culture, SOD $2^{-1-}$ neurons exhibit a profound reduction of neurite outgrowth and their mitochondria become fragmented and accumulate in the cell body. The structural abnormalities of the mitochondria are associated with reduced levels of phosphorylated (S637) dynamin related protein 1 (Drp1), a major mitochondrial fissionregulating protein, whereas mitochondrial fusion regulating proteins (OPA1 and MFN2) are relatively unaffected. Mitochondrial fission and Drp1 dephosphorylation coincide with impaired mitochondrial $\mathrm{Ca}^{2+}$ buffering capacity and an elevation of cytosolic $\mathrm{Ca}^{2+}$ levels. Treatment of $\mathrm{SOD}^{-1-}$ neurons with the $\mathrm{Ca}^{2+}$ chelator BAPTA-AM significantly increases levels of phosphorylated Drp1, reduces mitochondrial fragmentation and enables neurite outgrowth.
\end{abstract}

\section{Introduction}

Mitochondria play important roles in neurons including the generation of ATP that is essential for regulating excitability,

\section{Edited by N. Chandel}

These authors contributed equally: Qijin Zhao, Daoyuan Lu.

Electronic supplementary material The online version of this article (https://doi.org/10.1038/s41418-018-0230-4) contains supplementary material, which is available to authorized users.

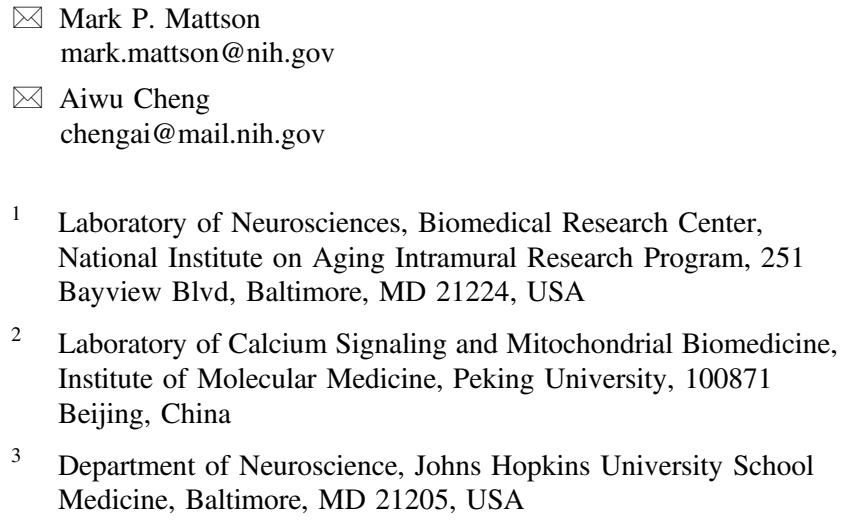

1 Laboratory of Neurosciences, Biomedical Research Center, National Institute on Aging Intramural Research Program, 251 Bayview Blvd, Baltimore, MD 21224, USA

2 Laboratory of Calcium Signaling and Mitochondrial Biomedicine, Institute of Molecular Medicine, Peking University, 100871 Beijing, China

3 Department of Neuroscience, Johns Hopkins University School Medicine, Baltimore, MD 21205, USA

$\mathrm{Ca}^{2+}$ and redox signaling, synaptic plasticity, and the arbitration of cell survival and death [1]. Mitochondria are highly dynamic organelles that can divide, fuse, and move within axons and dendrites. The number of mitochondria per cell increases during neuronal differentiation and synaptogenesis [2]. Previous studies suggest roles for mitochondrial dynamics in dendritic spine plasticity [3] and for mitochondrial biogenesis in the formation and plasticity of synapses in the developing and adult brain [2]. Treatment of neuroblastoma cells with chloramphenicol, an inhibitor of mitochondrial protein synthesis, prevents cell differentiation [4], and treatment of cultured embryonic hippocampal neurons with ethidium bromide, an agent that depletes mitochondrial DNA, prevents axon differentiation [5]. Mitochondrial dynamics in neural progenitor cells are also involved in the regulation of neurogenesis [6].

Mitochondria produce superoxide and hydrogen peroxide, reactive oxygen species (ROS) that may serve signaling functions [7]. However, excessive ROS generation can cause apoptotic and necrotic neuronal death, and are implicated in the pathogenesis of neurodegenerative disorders including Alzheimer's and Parkinson's diseases $[1,8]$. Mitochondria also play roles in the regulation of intracellular $\mathrm{Ca}^{2+}$ dynamics, and participate in many $\mathrm{Ca}^{2+}$-mediated signaling processes $[9,10]$. 
In neurons, mitochondria can buffer $\mathrm{Ca}^{2+}$ loads that occur during excitatory synaptic activity, and such mitochondrial $\mathrm{Ca}^{2+}$ uptake results in increased superoxide production [11]. Superoxide dismutases (SODs), including cytosolic and extracellular SODs (SOD1 and SOD3, respectively) and mitochondrial SOD (SOD2), are major antioxidant defense enzymes. Gene knockout studies in mice have shown that among the three SODs only SOD2 is essential for normal development and survival of mice. SOD2 deficient mice die at birth and exhibit severe anemia, neurodegeneration and myocardial damage [12-15]. Striatal neurons in mice with partial deficiency of SOD2 exhibit increased vulnerability to the mitochondrial toxins malonate and 3-nitroproponic acid [16]. On the other hand, the mitochondrial deacetylase SIRT3 increases SOD2 enzyme activity and protects neurons against excitotoxicity and mitochondrial toxins [17]. Moreover, overexpression of SOD2 reduces ischemic brain injury in a mouse stroke model [18] and ameliorates cognitive deficits in an Alzheimer's disease model [19]. Although it is clear that SOD2 and scavenging excessive mitochondrial ROS can protect neurons against excitotoxic and metabolic stress, roles for SOD2 in early events in neuronal development are unknown.

Previous studies of embryonic rat hippocampal neurons established key roles for $\mathrm{Ca}^{2+}$ in regulating the outgrowth of dendrites and the axon [20,21], and in the formation of glutamatergic synapses [22]. More recent studies have revealed roles for mitochondrial fission and fusion in the regulation of developmental and adult neuroplasticity $[1,3$, 23]. In the present study we found that neurite outgrowth is impaired and cell survival compromised in embryonic mouse cerebral cortical neurons lacking SOD2. The underlying molecular mechanism involves $\mathrm{Ca}^{2+}$ mediated dephosphorylation of the mitochondrial fission-regulating protein Drp1, mitochondrial fission and a failure of mitochondrial movement into nascent neurites. In the present study we show that by enhancing cellular $\mathrm{Ca}^{2+}$ handling and enabling normal mitochondrial dynamics, SOD2 is essential for the differentiation of embryonic cerebral cortical neurons.

\section{Results}

\section{SOD2 deficient embryonic cerebral cortical neurons exhibit altered mitochondrial morphology and reduced neurite outgrowth}

SOD2 homozygous knockout mice die during late embryonic development or shortly after birth $[15,24]$. To elucidate roles for SOD2 in neuronal development, we established primary neuronal cultures from embryonic day 14.5 SOD $2^{+/+}, \mathrm{SOD}_{2}^{+/-}$, and SOD $2^{-/-}$cerebral cortex. We measured the superoxide level in cultured cortical neurons using the superoxide-responsive fluorescent probe
MitoSOX Red. Levels of MitoSOX Red fluorescence were significantly greater in neurons lacking SOD2 compared to those in SOD2 ${ }^{+/+}$and SOD2 ${ }^{+/-}$neurons (Supplementary Fig. 1), consistent with the function of SOD2 as superoxide scavenger. Using Hoechst 33342 staining to quantify cell death (neurons with condensed and fragmented nuclei were considered dying or dead), approximately $90 \%$ of the initial number of $\mathrm{SOD}^{+/+}$and SOD2 ${ }^{+/-}$cortical neurons survived the first 3 days in culture and remained viable through culture day 7. Approximately $60 \%$ of the SOD $2^{-/-}$neurons survived during the first 3 days in culture and by culture day 7 essentially all of the SOD $2^{-/-}$neurons had died (Supplementary Fig. 2). SOD2 $2^{+/+}$and SOD2 $2^{+/-}$neurons extend multiple long neurites that express the neuronal marker beta-III tubulin (TuJ1) (Fig. 1a). In contrast, during the first several days in culture neurites in SOD $2^{-l-}$ neurons were markedly reduced in length and beta-III tubulin immunoreactivity accumulated in the cell body (Fig. 1a), To quantify the neurite length of individual neurons, we infected $\mathrm{SOD}^{+/+}$and SOD2 $2^{+/-}$neurons with an AAV-GFP for two days (1DIV to 3DIV) with a low viral titer that resulted in approximately $10 \%$ of the neurons expressing GFP, thereby enabling visualization of their neurites on a background of uninfected neurons (Fig. 1a, lower panels). The neurite length in 3DIV alive SOD2 ${ }^{-1-}$ neurons $(55.47 \pm 5.89 \mu \mathrm{m}$, $n=4$ mice) was less than $20 \%$ of the neurite length of $\mathrm{SOD}^{+/+}(383.62 \pm 20.39, n=4$ mice $)$ and $\mathrm{SOD}^{+/-}$ (326.57 18.24, $n=3$ mice) neurons (Fig. 1c).

We next evaluated mitochondrial morphology and numbers in $\mathrm{SOD} 2^{+/+}, \mathrm{SOD} 2^{+/-}$, and $\mathrm{SOD} 2^{-/-}$neurons on culture day 3. Neurons were loaded with Mitotracker green, a fluorescent mitochondrial stain which localizes to mitochondria in a mitochondrial membrane potential independent manner. Mitochondria in $\mathrm{SOD}^{+/+}$neurons were concentrated in the cell body and distributed throughout neurites wherein they exhibited a typical sausage-like morphology, and mitochondria in SOD2 $2^{+/-}$neurons were similarly distributed (Fig. 1b). In contrast, mitochondria in SOD $2^{-l-}$ neurons were located almost exclusively in the cell body where they appeared to be dotty, smaller and rounder than mitochondria in SOD2 ${ }^{+/+}$neurons (Fig. 1b). In neurites of both $\mathrm{SOD}^{+/+}$and $\mathrm{SOD} 2^{+/-}$neurons there were approximately 21-23 mitochondria per $200 \mu \mathrm{m}$, whereas there were only an average of 2 mitochondria per $200 \mu \mathrm{m}$ in neurites of SOD2 ${ }^{-1-}$ neurons (Fig. 1d).

\section{Embryonic cortical neurons lacking SOD2 exhibit reduced levels of the mitochondrial fission protein p-Drp1 (S637), without a change in levels of the mitochondrial fusion proteins Mfn2 and OPA1}

Mitochondrial fission is regulated by the protein Drp1; fission is inhibited when Drp1 is phosphorylated at Ser637 and 
Fig. 1 SOD2 deficiency results in reduced neurite outgrowth and impaired distribution of mitochondria into neurites of embryonic mouse cerebral cortical neurons. a The upper panels show images of cortical neurons from embryonic $\mathrm{SOD}^{+/+}, \mathrm{SOD}_{2}{ }^{+/-}$, and $\mathrm{SOD} 2^{-l-}$ mice (maintained in $20 \% \mathrm{O}_{2}$ ) at 3 days in culture that were fixed and immunostained using a Tuj1 antibody (green) and counterstained with DAPI to label nuclear DNA (blue). The lower two panels show confocal images of individual GFP ${ }^{+}$ neurons $48 \mathrm{~h}$ after transfection with a GFP plasmid illustrating the detailed morphology of neurons. Bar $=50 \mu \mathrm{m}$. b Confocal images of Mitotracker green fluorescence in SOD2 ${ }^{+/+}$, $\mathrm{SOD} 2^{+--}$, and SOD $2^{-1-}$ cortical neurons at 3 days in culture. For SOD2 ${ }^{-l-}$ neurons, a merged image of phase-contrast and Mitotracker green is also shown (upper right). The lower panels show higher magnification examples of mitochondria in neurites. Bar $=50 \mu \mathrm{m}$. c, d Results of measurements of total neurite length (c) and mitochondria numbers per 200 $\mu \mathrm{m}$ neurite length $(\mathbf{d})$. Values are mean \pm SEM $(n=3-4$ separate culture preparations). $* * * p<$ 0.001. (ANOVA with student Newman-Keuls post-hoc tests)

A
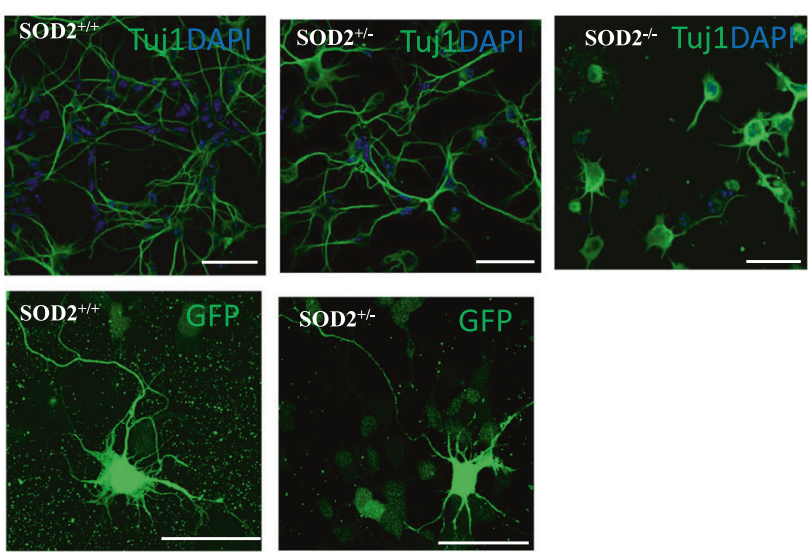

B
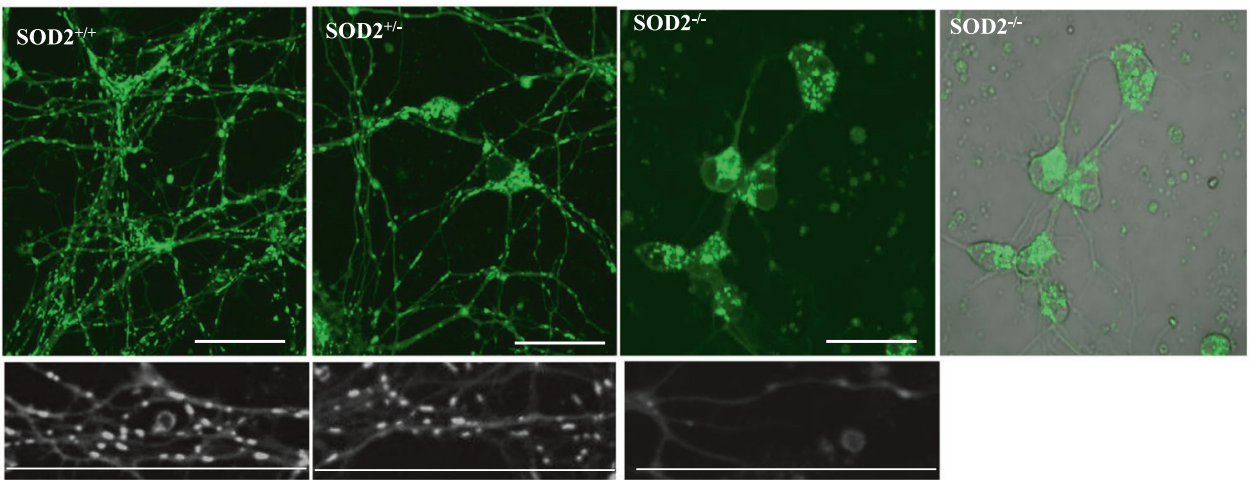

$\mathrm{C}$

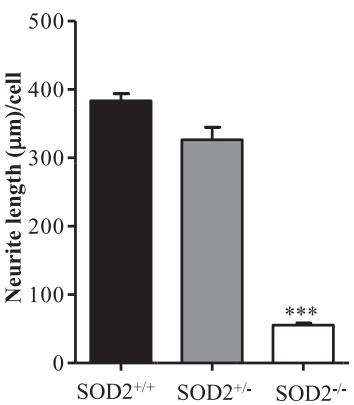

D

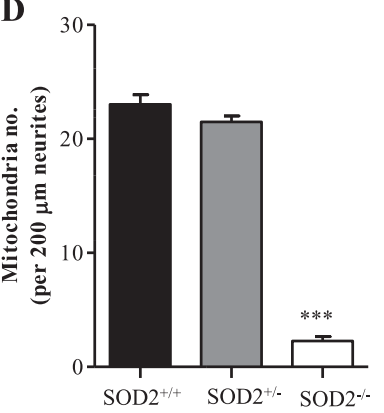

dephosphorylation at Ser637 by the $\mathrm{Ca}^{2+}$-activated phosphatase calcineurin reverses this inhibition [25, 26]. Immunoblot analysis demonstrated that on culture day 3, the levels of phosphorylated Drp1 (at Ser637) and total Drp1 were significantly reduced (by $\sim 75$ and $55 \%$, respectively) in SOD2 ${ }^{-1-}$ neurons compared to SOD2 ${ }^{+/+}$and SOD2 ${ }^{+/-}$neurons (Fig. 2b). There were no differences in levels of Drp1 and pDrp1 between SOD $2^{+/+}$and SOD $2^{+/}$neurons. On culture day 2, levels of p-Drp1 (Ser637) were significant reduced (by $\sim 40 \%$ ) in neurons from SOD2 ${ }^{-1-}$ mice, whereas total Drp1 levels were not altered compared to Drp1 levels in SOD2 ${ }^{+/+}$ and SOD $2^{+/-}$neurons (Fig. 2a), indicating a progressive disinhibition of mitochondrial fission. The fusion of mitochondria is mediated by homophilic and heterophilic interactions of cytosolic domains of mitofusins (Mfn1 and Mfn2) which are proteins located in the outer mitochondrial membrane, and OPA1 which is located in the inter-membrane space. There were no significant differences in levels of Mfn2 and OPA1 in cerebral cortical neurons from $\mathrm{SOD} 2^{+/+}, \mathrm{SOD} 2^{+/-}$and $\mathrm{SOD} 2^{-/-}$mice on culture day 3 (Fig. 2c). Levels of the voltage-dependent anion carrier protein (VDAC) was not altered in SOD $2^{-/-}$neurons compared to that of SOD $2^{+/+}$and SOD $2^{+l-}$ neurons (Fig. 2b), suggesting that the total mitochondrial mass/cell was unaffected by SOD2 deficiency.

\section{$\mathrm{Ca}^{2+}$ mediates Drp1 dephosphorylation in developing cerebral cortical neurons}

Previous studies have shown that the phosphorylation of Drp1 at Ser637 is a critical regulatory modification, with 
A

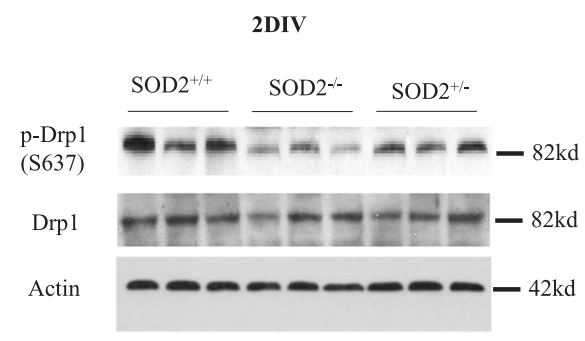

B

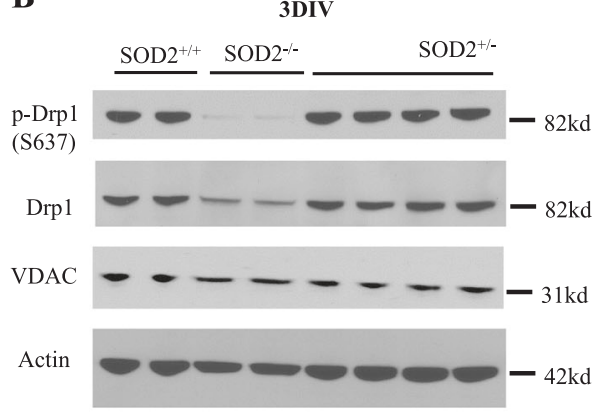

C

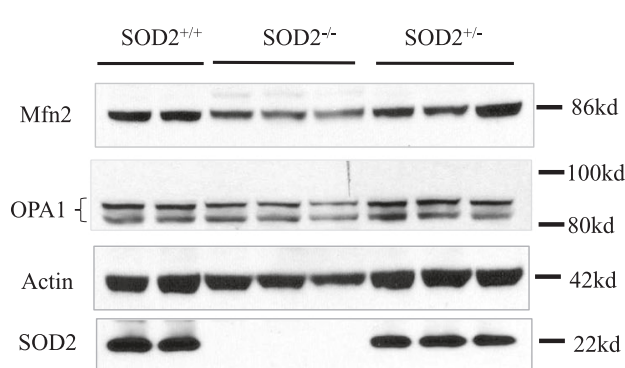

Fig. 2 SOD2 deficiency results in a reduction in levels of p-Drp1 (S637) in cerebral cortical neurons. a, b Immunoblot analysis of phosphorylated Drp1 (p-Drp1) and total Drp1 in cerebral cortical neurons from SOD2 ${ }^{+/+}, \mathrm{SOD} 2^{+/-}$, and $\mathrm{SOD} 2^{-/-}$mice at 2 days (a) and 3 days (b) in culture. The blots were re-probed to with antibodies either against VDAC or actin. The right panels are the results of densitometric analysis of blots showing p-Drp1, Drp and VDAC protein levels (percentage change compared to the value for $\left.\mathrm{SOD} 2^{+/+}\right)$. Values are mean $\pm \mathrm{SD}(n=3-7$ cultures from separate

cAMP-dependent protein kinase phosphorylating Ser637 [27] and the $\mathrm{Ca}^{2+}$-calmodulin-dependent protein phosphatase calcineurin dephosphorylating Ser637 [26]. Dephosphorylation of Drp1 at Ser637 by calcineurin results in translocation of Drp1 from the cytosol to the mitochondrial membrane [26]. We therefore performed experiments to characterize the effects of $\mathrm{Ca}^{2+}$ influx on Drp1 phosphorylation and mitochondrial fission in mouse cerebral cortical neurons. Both cytoplasmic and intramitochondrial $\mathrm{Ca}^{2+}$ concentrations increase transiently in mouse cortical neurons in response to activation of glutamate receptors [17]. Using a mitochondrially targeted $\mathrm{Ca}^{2+}$-sensitive fluorescent probe
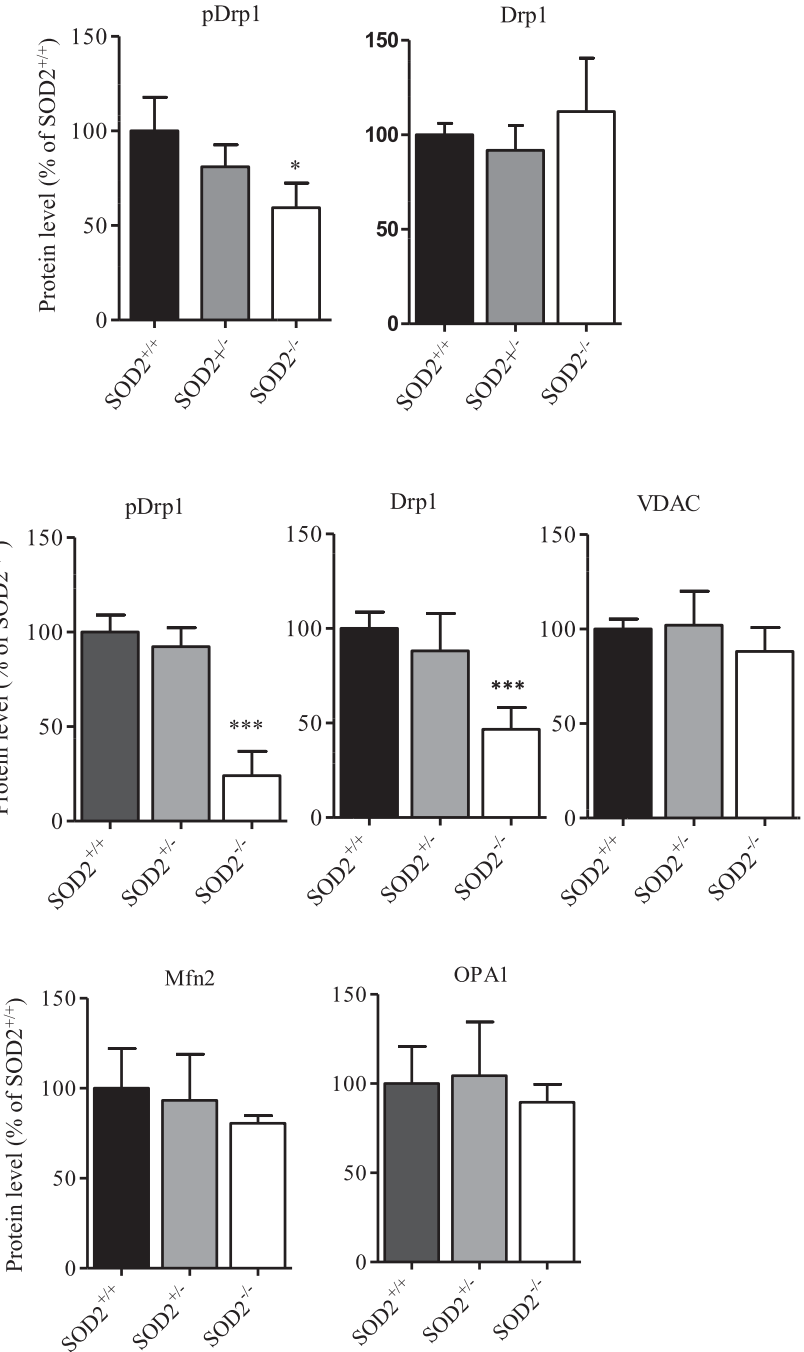

embryos). $* p<0.05, * * * p<0.001$ (ANOVA with student NewmanKeuls post-hoc tests). c Immunoblot analysis of Mfn2 and OPA1 in $\mathrm{SOD} 2^{+/+}, \mathrm{SOD}^{+/-}$, and SOD $2^{-/-}$cortical neurons $(3$ days in culture). The blots were re-probed with antibodies either against SOD2 or actin. The right panels are the results of densitometric analysis of blots showing Mfn2 and OPA1 protein levels (percentage change compared to the value of $\left.\mathrm{SOD}^{+/+}\right)$. Values are mean $\pm \mathrm{SD}(n=4-6$ cultures from separate embryos)

(mito-GCaMP3), we found that the intramitochondrial $\mathrm{Ca}^{2+}$ concentration increased rapidly in response to $10 \mu \mathrm{M}$ glutamate, peaking within a few seconds and then decreasing but remaining elevated compared to the baseline $\mathrm{Ca}^{2+}$ concentration during a $5 \mathrm{~min}$ imaging period (Fig. 3a, b). The average mitochondrial length was significantly reduced, and the number of mitochondria/neuron was significantly increased in response to glutamate (Fig. 3c), suggesting mitochondrial fission. To determine the effects of glutamate on phosphorylation of Drp1 at Ser637, we exposed cortical neurons to increasing concentrations of glutamate and then evaluated p-Drp1 (Ser637) and Drp1 levels by immunoblot 
Fig. 3 Cytosol calcium elevation causes mitochondrial fission and Drp1 (S637) de-

phosphorylation. a Images showing mitochondrial morphology of neurons expressing the mitochondrial targeted calcium indicator GCaMP3 (mito-GCaMP3) before and after $(3.2 \mathrm{~s}$ and $133 \mathrm{~s})$ exposure to glutamate $(10 \mu \mathrm{M})$. The red box shows examples of fragmented mitochondria. b Mitochondrial $\mathrm{Ca}^{2+}$

concentrations were measured by imaging of mito-GCaMP3, prior to and during exposure of cortical neurons to $10 \mu \mathrm{M}$ glutamate ( $n=6$ neurons for the trace). c Mitochondrial sizes and numbers before and $5 \mathrm{~min}$ after glutamate treatment $(n=6, * * p$ $<0.001)$. d, e Immunoblot analysis of p-Drp1 (S637) and Drp1 before (Ctr) and 5 min after glutamate treatment at the indicated concentrations $(1,10$, $100,500 \mu \mathrm{M})(\mathbf{d})$, and at increasing time points after exposure to $10 \mu \mathrm{M}$ glutamate (5 min, $30 \mathrm{~min}, 2 \mathrm{~h}$ and $4 \mathrm{~h}$ ) (e).

The blots were re-probed with an antibody against actin. The right panels are the results of densitometric analysis of blots showing p-Drp1 and Drp1 levels (percentage change compared to the control value). Values are mean $\pm \mathrm{SD}(n=6$ separate cultures). $* * p<0.01$ compared to the control value (ANOVA with student Newman-Keuls post-hoc tests). f Immunoblot and densitometric analysis of $\mathrm{p}$ Drp1 (S637) and Drp1 before and $5 \mathrm{~min}$ after exposure to 10 $\mu \mathrm{M}$ glutamate in the cultures that were pretreated with BAPTAAM $(10 \mu \mathrm{M})$, FK506 $(10 \mathrm{nM})$, okadaic acid (OA) (10 nM).

NPT, no pretreatment. Values are mean $\pm \mathrm{SD}(n=8$ separate cultures), $* * p<0.01$ (ANOVA with student Newman-Keuls post-hoc tests)
A
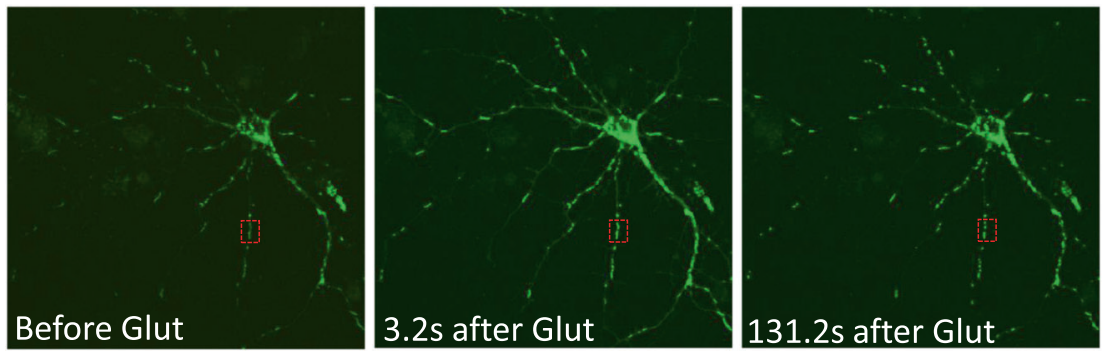

B
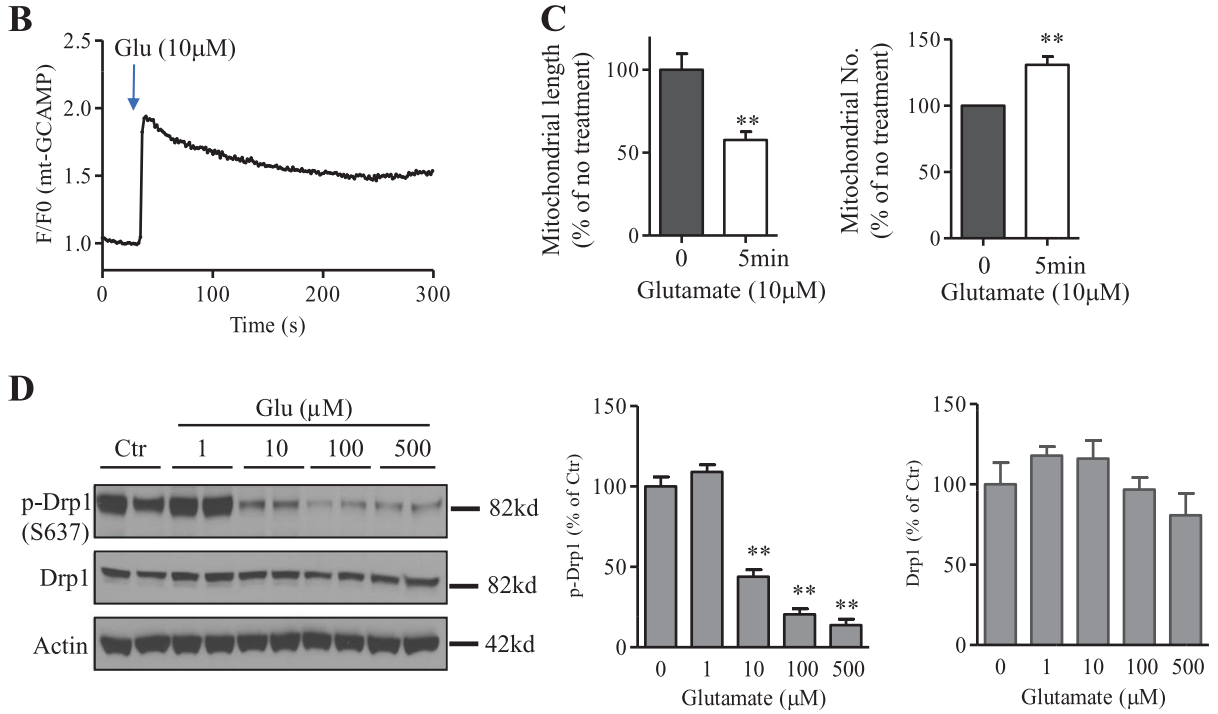

$\mathbf{E}$
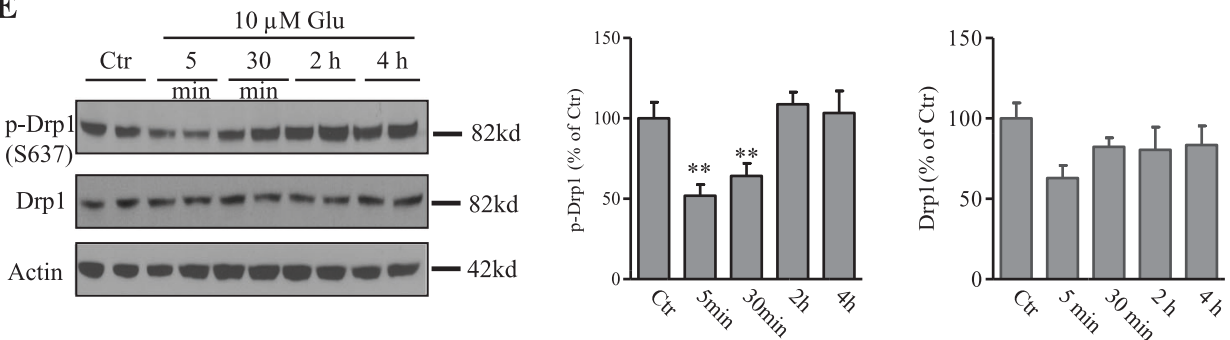

F
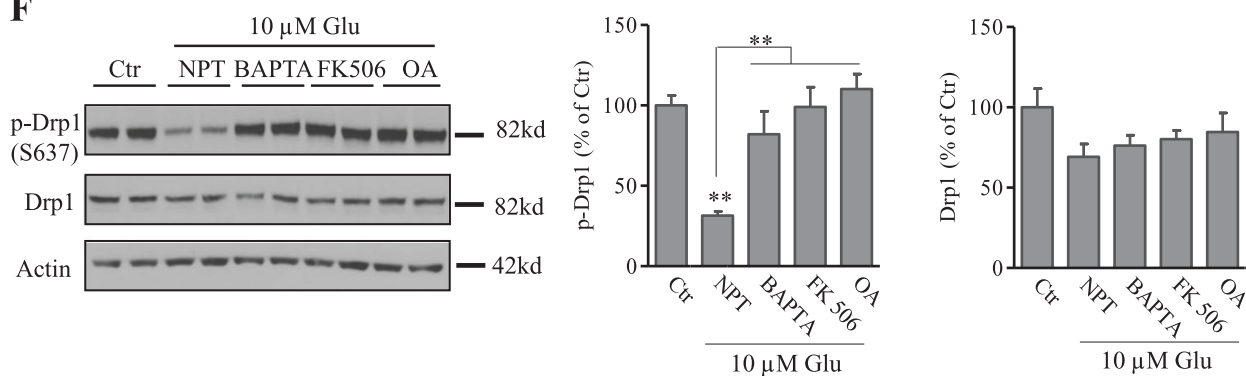

analysis of cell lysates. The levels of p-Drp1 were greatly reduced during a $5 \mathrm{~min}$ exposure to glutamates at 10,100 , $500 \mu \mathrm{M}$, in a concentration dependent manner (Fig. 3d). The reduction in p-Drp1 levels in response to exposure to $10 \mu \mathrm{M}$ glutamate was transient, remaining reduced through $30 \mathrm{~min}$ and then recovering to baseline levels by $2 \mathrm{~h}$ (Fig. 3e). The amount of total Drp1 was unchanged in neurons exposed to glutamate (Fig. 3d, e). Pretreatment of cultures with the cytoplasmic $\mathrm{Ca}^{2+}$ chelator BAPTA-AM $(10 \mu \mathrm{M})$, the calcineurin inhibitor FK506 or the protein phosphatase $2 \mathrm{~A}$ inhibitor okadaic acid (OA) prevented dephosphorylation of Drp1 at ser637 in neurons exposed to glutamate (Fig. 3f).

To further investigate whether glutamate-induced dephosphorylation of Drp1 (S637) indeed targets Drp1 to 
Fig. 4 Drp1 translocates to mitochondria in response to glutamate stimulation. a Confocal images showing Drp1 immunoreactivity (green) and COX1 immunoreactivity (red) in cortical neurons from embryonic SOD $2^{+/+}$mice (upper two rows of images) and SOD2 $2^{-1-}$ mice (lower row) at 3 days in culture. The cells were counterstained with DAPI to label nuclear DNA (blue). The SOD2 $2^{+/+}$cortical neurons were treated for $5 \mathrm{~min}$ with either vehicle (upper panels) or glutamate (Glut:100 $\mu \mathrm{M}$; middle panels) or vehicle. Bar $=20 \mu \mathrm{m}$. The arrowheads indicate the Drp $1^{-} \mathrm{COX} 1^{+}$ puncta and arrows indicate Drp1 ${ }^{+} \mathrm{COX}^{+}$puncta. $\mathbf{b}$ Results of quantification of percentages of the total number of COX1 positive puncta in neurites that are also Drp1 positive puncta in control (Ctr) cultures and cultures exposed to the indicated concentrations of glutamate (Glut) for 5 min. c Results of quantification of percentages of the total number of COX1 positive puncta in neurites that are also Drp1 positive in neurons from SOD2 ${ }^{+/+}$and SOD2 $2^{-/-}$ mice at 3 days in culture. Values are mean $\pm \mathrm{SD}(n=3$ cultures from separate animals). $* * p<$ 0.001. (ANOVA with student Newman-Keuls post-hoc tests or student $\mathrm{t}$ test)
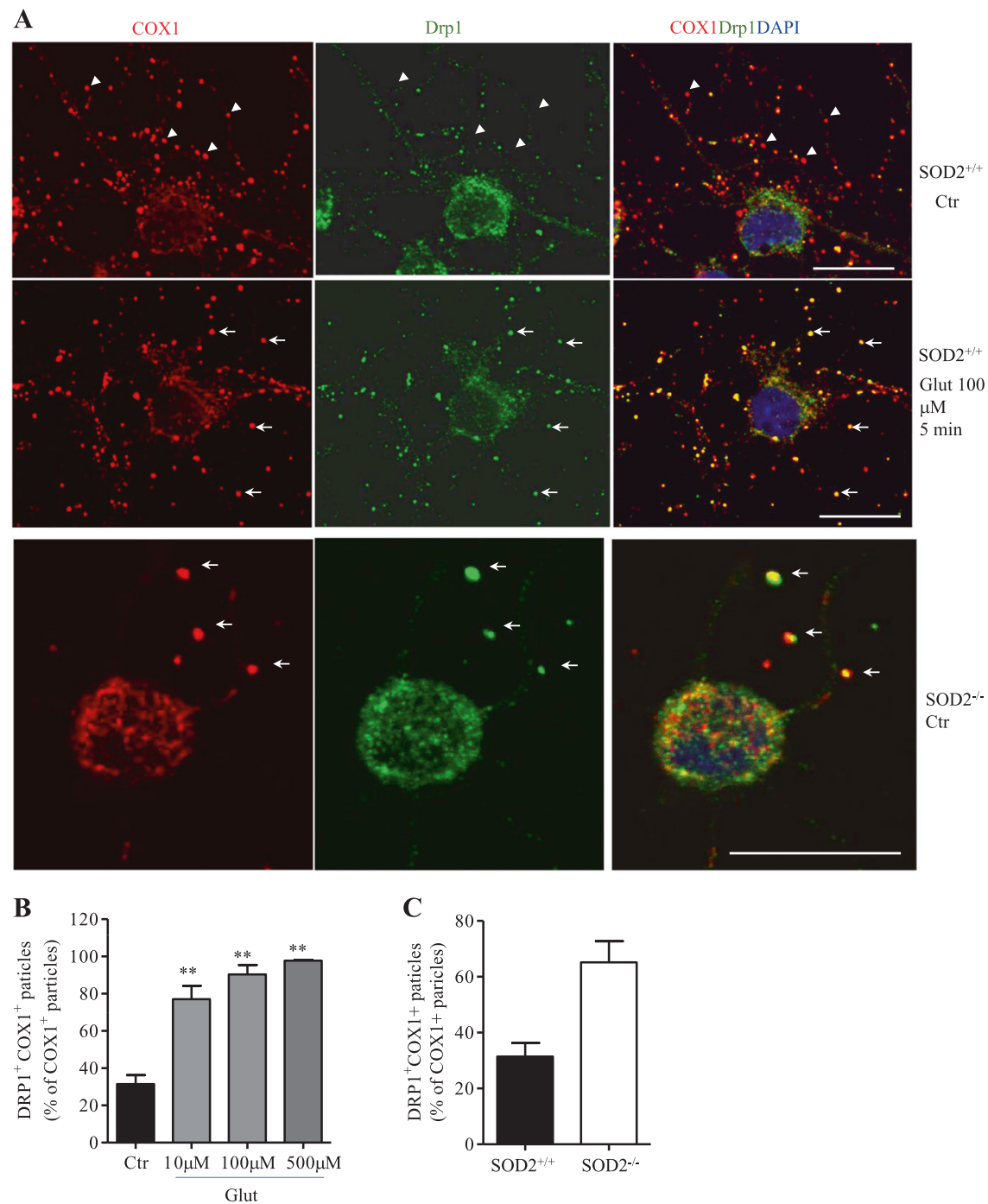

mitochondria, we performed double-label immunostaining with a Drp1 antibody and an antibody against the mitochondrial protein COX1 in cultured cortical neurons that were treated for $5 \mathrm{~min}$ with either glutamate or vehicle control. As expected, in control $\mathrm{SOD}^{+/+}$neurons COX1 immunoreactivity was localized in discrete puncta distributed along neurites and in perinuclear cytoplasm of the cell body consistent with a mitochondrial localization, and approximately $30 \%$ of the mitochondria exhibited strong particle-like Drp1 immunoreactivity whereas the rest of mitochondria $(\sim 70 \%)$ exhibited little or no Drp1 immunoreactivity (Fig. 4a, b). In contrast, in neurons lacking SOD2 more than $60 \%$ of the COX1 immunoreactive puncta co-localized with strong Drp1 staining (Fig. 4a, lower panel). Strikingly, within $5 \mathrm{~min}$ of exposure of $\mathrm{SOD}^{+/+}$ neurons to glutamate $(10-500 \mu \mathrm{M})$ treatment the percentage of COX1 immunoreactive puncta that exhibited strong Drp1 immunoreactivity increased to more than $80 \%$ (Fig. 4a, b). These data suggest that glutamate elevates intracellular
$\mathrm{Ca}^{2+}$ levels which, in turn results in Drp1 dephosphorylation and translocation to mitochondria.

\section{Cytoplasmic $\mathrm{Ca}^{2+}$ concentration is elevated and mitochondrial $\mathrm{Ca}^{2+}$ handling is impaired in SOD2- deficient neurons}

To quantify the cytoplasmic free $\mathrm{Ca}^{2+}$ concentration $\left(\left[\mathrm{Ca}^{2+}\right]_{\text {cyt }}\right)$ we performed confocal imaging of neurons loaded with calcium indicators Fluo-3 and Fura-red and determined the ratio Fluo-3/Fura-red as described previously [28-30]. The red curve in the graph shown in Fig. 5a is the result of fitting the dual indicator fluorescence ratio $(R)$ to the equation where different $\mathrm{Ca}^{2+}$ concentration values are used, and the fitting yielded $\mathrm{K}_{\mathrm{d}} 1=1396.8 \mathrm{nM}$ and $\mathrm{K}_{\mathrm{d}} 2=343 \mathrm{nM}$ for Fluo 3 and Fura red, respectively in cultured cells. The apparent increase of $\mathrm{K}_{\mathrm{d}}$ value in cells compared with those in vitro has been reported previously $[31,32]$ and is attributable to the intracellular milieu 

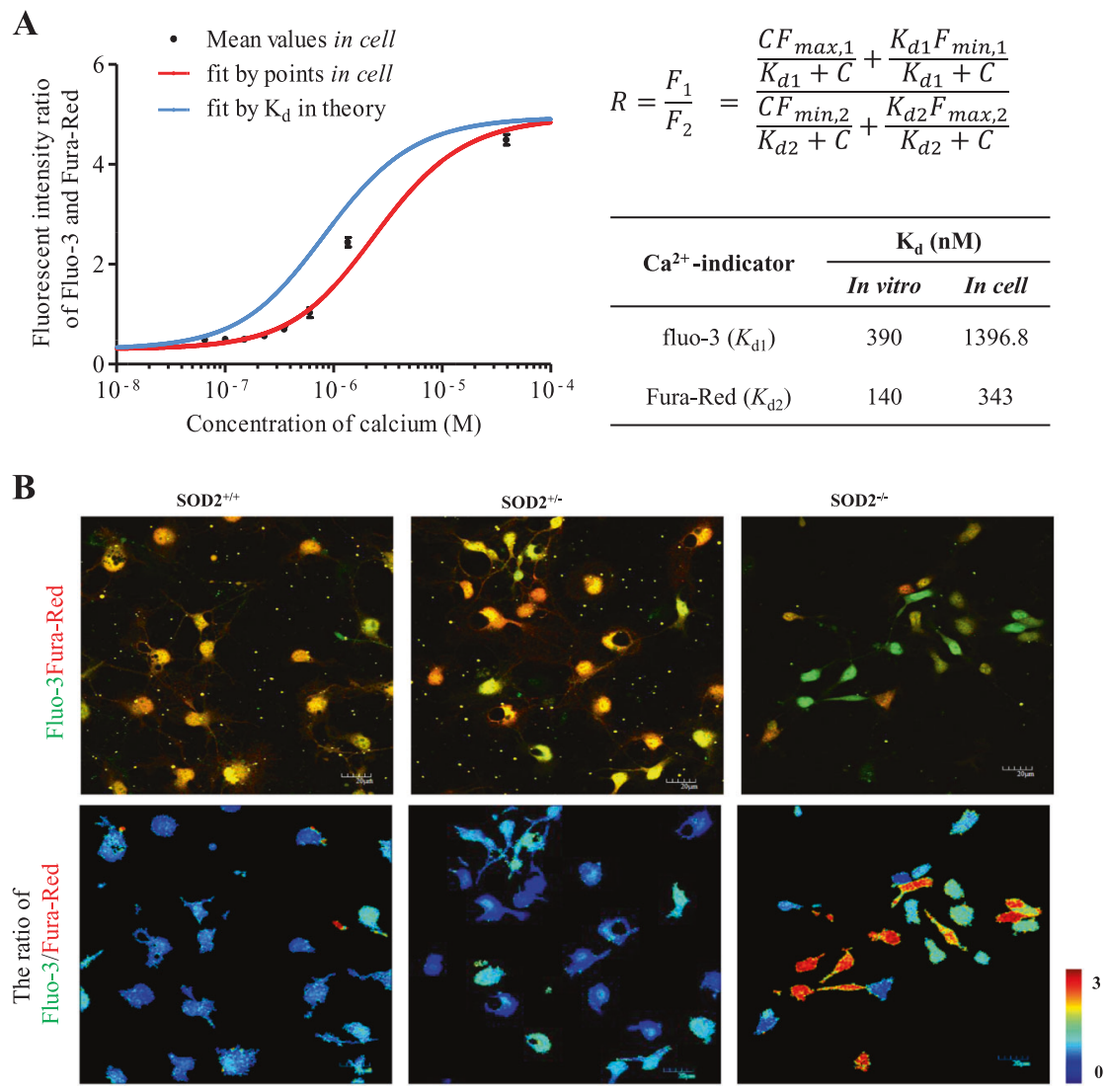

C
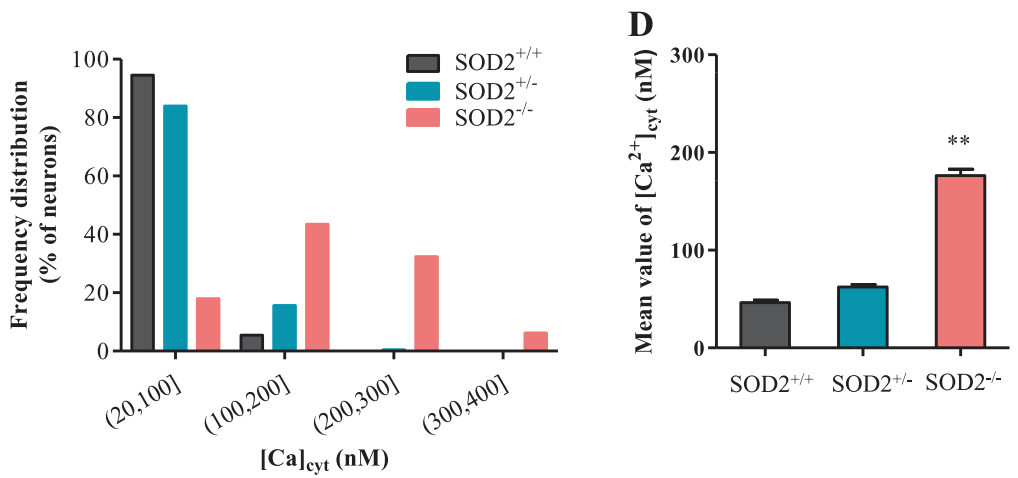

Fig. 5 The cytosolic calcium concentration is significantly elevated in $\mathrm{SOD} 2^{-/-}$neurons compared with $\mathrm{SOD} 2^{+/+}$and SOD2 ${ }^{+/-}$neurons. a In cell calibration of Fluo-3 and Fura-Red as a ratiometric $\left[\mathrm{Ca}^{2+}\right]$ measurement pair. The black dots are data from a calibration of cytosolic calcium concentration $\left[\mathrm{Ca}^{2+}\right]_{\text {cyt }}$ and the fluorescence intensity ratio of Fluo-3 $(1 \mu \mathrm{M}) /$ Fura-Red $(5 \mu \mathrm{M})$ in primary cultured embryonic cortical neurons. Values are mean \pm SEM. $(n=35-44$ neurons for each point). The blue line is the fitting curve by $\mathrm{Kd}$ in theory and the red line is the fitting curve of the points by fitting the dual indicator fluorescence ratio $(\mathrm{R})$ and calcium concentration to the $\mathrm{Ca}^{2+}$ calculation equation (right). The Kd in cells determined by curve

affecting the properties of dyes such as indicator-protein interaction in cells. Therefore, using the fitting curve (red line) as the $\left[\mathrm{Ca}^{2+}\right]_{\text {cyt }}$ calibration curve was more accurate than the curve predicted based on the in vitro $\mathrm{Ca}^{2+}$ dissociation constants of Fluo-3 and Fura-red (blue line) fitting is shown in comparison to the theoretical $\mathrm{Kd}$ (or in vitro $\mathrm{Kd}$ ) (right table). b Confocal images (upper panels) showing the merged images of Fluo-3 (green) and Fura-red (red) and the ratio (Fluo3/Furared) images with $\left[\mathrm{Ca}^{2+}\right]_{\text {cyt }}$ represented in pseudocolor (lower panels) after $30 \mathrm{~min}$ loading in SOD2 ${ }^{+/+}, \mathrm{SOD} 2^{+/-}$, and $\mathrm{SOD} 2^{-/-}$cortical neurons at 2 days in culture. $\mathrm{Bar}=20 \mu \mathrm{m}$. The range of the Fluo3/ Fura-red ratios in the color scale bar is from 0 to 3 . c The $\left[\mathrm{Ca}^{2+}\right]_{\text {cyt }}$ frequency distributions among neurons analyzed: $\operatorname{SOD} 2^{+/+}(n=164)$; $\mathrm{SOD}^{+l-}(n=224)$; and SOD2 ${ }^{-/-}(n=145)$ cortical neurons from $3-$ 4 mice. $\mathbf{d}$ the average values of resting $\left[\mathrm{Ca}^{2+}\right]_{\text {cyt. }}$ Values are mean \pm SD. $* * p<0.01$ compared to the value for $\mathrm{SOD}^{+i+}$ neurons

(Fig. 5a). When SOD2 $2^{+/+}$, SOD2 $2^{+/-}$, SOD $2^{-/-}$neurons at culture day 2 were loaded for $30 \mathrm{~min}$ with Fluo-3 $(1 \mu \mathrm{M})$ and Fura-red $(5 \mu \mathrm{M})$, and confocal images of neurons were acquired using identical parameters, SOD $2^{-/-}$neurons exhibited more intense Fluo-3 fluorescence (green) and 

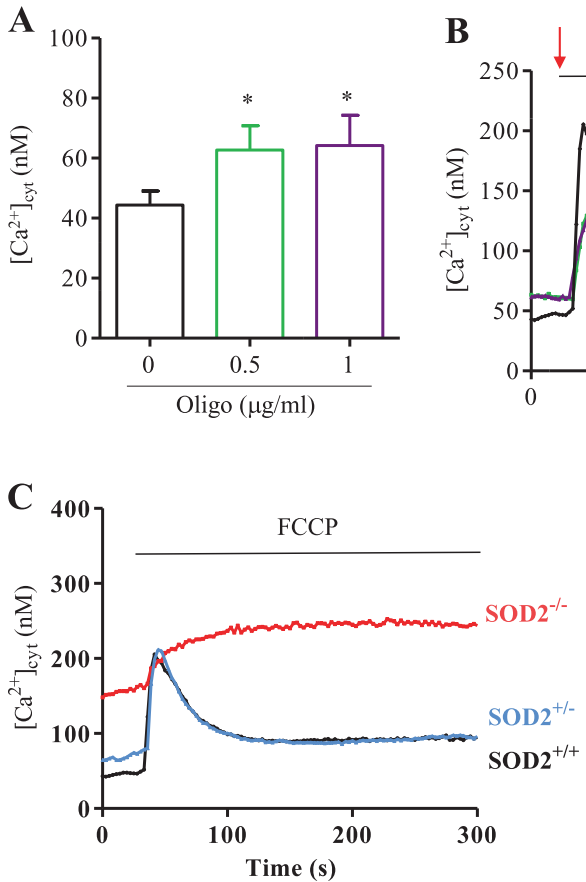
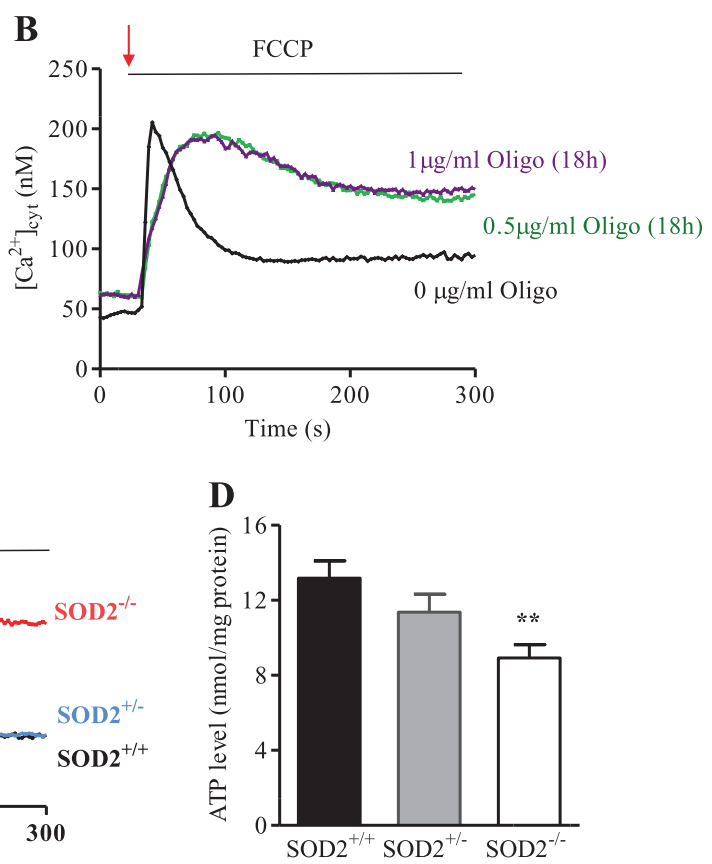

Fig. 6 SOD2 deficiency perturbs cellular $\mathrm{Ca}^{2+}$ handling and reduces ATP production in embryonic cortical neurons. a Resting $\left[\mathrm{Ca}^{2+}\right]_{\mathrm{cyt}}$ in cultured $S O D 2^{+/+}$cortical neurons after $18 \mathrm{~h}$ treatment with $0,0.5$, or $1 \mu \mathrm{g} / \mathrm{ml}$ oligomycin (oligo). Values are mean \pm SEM. $* p<0.05$. b The $\left[\mathrm{Ca}^{2+}\right]_{\text {cyt }}$ prior to and during exposure of $S O D 2^{+/+}$cortical neurons to $2 \mu \mathrm{M}$ FCCP without or with $18 \mathrm{~h}$ pretreatment with 0.5 or $1 \mu \mathrm{g} / \mathrm{ml}$ oligomycin (oligo). Arrowheads mark the time of FCCP stimulation. c Cytosolic $\mathrm{Ca}^{2+}$ transients induced by exposure of neurons to the

reduced Fura-red fluorescence (red) compared to SOD2 $2^{+/+}$ and SOD2 $2^{+-}$neurons (Fig. 5b upper panels), suggesting that the $\left[\mathrm{Ca}^{2+}\right]_{\text {cyt }}$ was elevated in the SOD2 $2^{-/-}$neurons. Visual examination of Fluo-3/Fura-red ratiometric images revealed an obvious elevation of $\left[\mathrm{Ca}^{2+}\right]_{\text {cyt }}$ in $\mathrm{SOD} 2^{-1-}$ neurons (Fig. 5b lower panels) which was confirmed by quantitative analysis (Fig. 5 c, d). Examination of $\left[\mathrm{Ca}^{2+}\right]_{\mathrm{cyt}}$ in individual cells showed that the $\left[\mathrm{Ca}^{2+}\right]_{\mathrm{cyt}}$ was low in neurons on culture day 2 with a mean value of $46.5 \mathrm{nM}$ and a standard deviation (SD) of $30.9 \mathrm{nM}(n=164)$. There was a large variability of $\left[\mathrm{Ca}^{2+}\right]_{\mathrm{cyt}}$ among $\mathrm{SOD} 2^{-/-}$neurons with a clear shift toward higher $\left[\mathrm{Ca}^{2+}\right]_{\text {cyt }}$ (Fig. 5 c, d). The average resting $\left[\mathrm{Ca}^{2+}\right]_{\text {cyt }}$ for neurons from the three SOD genotypes were: $46.5 \pm 30.9 \mathrm{nM}(n=164)$ for $\mathrm{SOD} 2^{+/+}$, $62.4 \pm 35.2 \mathrm{nM} \quad(n=224)$ for $\mathrm{SOD} 2^{+/-}$and $176.4 \pm$ $76.5 \mathrm{nM}(n=145)$ for SOD $2^{-l-}$ neurons (Fig. 5d). Thus, there were large $300-350 \%$ increases in resting $\left[\mathrm{Ca}^{2+}\right]_{\mathrm{cyt}}$ in $\mathrm{SOD} 2^{-/-}$neurons compared with $\mathrm{SOD} 2^{+/+}$and SOD2 $2^{+/-}$ neurons.

We next determined the impact of SOD2 deficiency on mitochondrial uncoupling-induced $\left[\mathrm{Ca}^{2+}\right]_{\mathrm{cyt}}$ dynamics in cortical neurons. When we treated neurons on culture day 2 with the mitochondrial ATP synthase inhibitor oligomycin $(0.5$ or $1 \mu \mathrm{g} / \mathrm{ml})$ for $18 \mathrm{~h}$, the $\mathrm{SOD}^{+/+}$and $\mathrm{SOD} 2^{+/-}$ mitochondrial un-coupler FCCP. The $\left[\mathrm{Ca}^{2+}\right]_{\text {cyt }}$ was measured by imaging prior to and during exposure of $\mathrm{SOD} 2^{+/+}, \mathrm{SOD}^{+/-}$, and SOD $2^{-I-}$ cortical neurons ( 2 days in culture) to $2 \mu \mathrm{M}$ FCCP. In both c and d, each trace is the mean value of approximately $25-50$ cells. d ATP levels (normalized to cellular protein levels) in primary cultured $\mathrm{SOD} 2^{+/+}, \mathrm{SOD}_{2}^{+/-}$, and $\mathrm{SOD} 2^{-/-}$cortical neurons $(2$ days in culture). Values are mean $\pm \operatorname{SEM}(n=4-5$ mice $)$. ${ }^{* *} p<0.01$ compared to the value for $\mathrm{SOD}^{+/+}$neurons

neurons remained alive, whereas $\mathrm{SOD} 2^{-1-}$ neurons all died (data not shown). We then exposed SOD2 $2^{+/+}$neurons to oligomycin $(0.5 \mu \mathrm{g} / \mathrm{ml})$ and measured $\left[\mathrm{Ca}^{2+}\right]_{\mathrm{cyt}} 18 \mathrm{~h}$ later. Oligomycin treatment significantly increased $\left[\mathrm{Ca}^{2+}\right]_{\text {cyt }}(0.5$ $\mu \mathrm{g} / \mathrm{ml} ; 62.7 \pm 42.9 \mathrm{nM}, n=41) ; 1.0 \mu \mathrm{g} / \mathrm{ml}, 64.2 \pm 46.5 \mathrm{nM}$, $n=29)$ compared with that of control cultures $(44.4 \pm 26.7$, $n=65$ ) (Fig. 6a). Thus, the $\left[\mathrm{Ca}^{2+}\right]_{\text {cyt }}$ was elevated by $\sim 40 \%$ in oligomycin-treated neurons. Neurons were then loaded with Fluo- 3 and Fura-red and the $\left[\mathrm{Ca}^{2+}\right]_{\text {cyt }}$ was measured before and during exposure of the neurons to the mitochondrial uncoupling agent FCCP. In neurons not treated with oligomycin the $\left[\mathrm{Ca}^{2+}\right]_{\text {cyt }}$ rose rapidly in response to FCCP and then recovered towards baseline $\left[\mathrm{Ca}^{2+}\right]_{\mathrm{i}}$, within 2 min (Fig. 6b). The $\left[\mathrm{Ca}^{2+}\right]_{\text {cyt }}$ response to FCCP in oligomycin-treated neurons reached a peak concentration similar to that of vehicle-treated neurons, but thereafter remained elevated (Fig. 6b). The values for time to peak $\left[\mathrm{Ca}^{2+}\right]_{\text {cyt }}$, peak $\left[\mathrm{Ca}^{2+}\right]_{\text {cyt }}$, plateau $\left[\mathrm{Ca}^{2+}\right]_{\text {cyt }}$ and "half-life" are shown in Table 1 . We next measured $\left[\mathrm{Ca}^{2+}\right]_{\text {cyt }}$ responses to FCCP in neurons from $\mathrm{SOD} 2^{-/-}$, SOD $2^{+/-}$, and $\mathrm{SOD} 2^{+/+}$mice and found that $\mathrm{SOD} 2^{+/+}$and SOD $2^{+/-}$ neurons exhibited similar $\left[\mathrm{Ca}^{2+}\right]_{i}$ responses (Fig. 6c). Consistent with results above (Fig. 4), the basal $\left[\mathrm{Ca}^{2+}\right]_{\mathrm{cyt}}$ was significantly greater in SOD2 $2^{-1-}$ neurons compared to 
Table 1 Cytosolic $\mathrm{Ca}^{2+}$ responses to the mitochondrial uncoupling agent FCCP in cultured cerebral cortical neurons from $\mathrm{SOD}^{+1+}$ $\mathrm{SOD}^{+/-}$and $\mathrm{SOD}^{-1-}$ neurons

\begin{tabular}{llll}
\hline Parameters & SOD2 $^{+/+}$ & SOD2 $^{+/+}+0.5 \mu \mathrm{g} / \mathrm{ml}$ oligo & SOD2 $^{+/+}+1 \mu \mathrm{g} / \mathrm{ml}$ oligo \\
\hline Time to peak $\left(\mathrm{T}_{\mathrm{p}}\right)(\mathrm{s})$ & 8.326 & $61.141^{* * *}$ & $63.906^{* * *}$ \\
Peak value $\left(\triangle \mathrm{C} / \mathrm{C}_{0}\right)$ & 2.86 & $2.18^{* * *}$ & $2.17 * * *$ \\
Plateau $(\mathrm{nM})$ & 91.83 & $142.6 * * *$ & $147.8^{* * *}$ \\
Half life $\left(\mathrm{t}_{1 / 2}\right)(\mathrm{s})$ & 17.13 & 37.93 & 37.65
\end{tabular}

\begin{tabular}{llll}
\hline Parameters & SOD2 $^{+/+}$ & SOD2 $^{+/-}$ & SOD2 $^{-/-}$ \\
\hline Time to peak $\left(\mathrm{T}_{\mathrm{p}}\right)(\mathrm{s})$ & 8.326 & 9.034 & $75.03^{* * * *}$ \\
Peak value $\left(\triangle \mathrm{C} / \mathrm{C}_{0}\right)$ & 2.86 & 2.29 & $0.73^{* * *}$ \\
Plateau $(\mathrm{nM})$ & 91.83 & 92.2 & $254.5^{* * *}$ \\
Half life $\left(\mathrm{t}_{1 / 2}\right)(\mathrm{s})$ & 17.13 & 14.52 & \\
\hline
\end{tabular}

Upper-Kinetic analysis of FCCP treatment response in SOD2 ${ }^{+1+}$ neurons with and without treatment with oligomycin (oligo). Lower-Kinetic analysis of FCCP treatment response in SOD2 ${ }^{+/+}, \mathrm{SOD} 2^{+/-}, \mathrm{SOD} 2^{-/-}$ neurons. $* * * P<0.001$
$\mathrm{SOD} 2^{+/+}$neurons and, in response to FCCP the $\left[\mathrm{Ca}^{2+}\right]_{\text {cyt }}$ in $\mathrm{SOD} 2^{-1-}$ neurons rose slowly and remained elevated for at least $5 \mathrm{~min}$ (Fig. 6c). The values for time to peak $\left[\mathrm{Ca}^{2+}\right]_{\text {cyt }}$, peak $\left[\mathrm{Ca}^{2+}\right]_{\text {cyt }}$, plateau $\left[\mathrm{Ca}^{2+}\right]_{\text {cyt }}$ and "half-life" are shown in Table 1. We found that cellular ATP levels were significantly lower in cultured SOD $2^{-1-}$ neurons compared to SOD $2^{+/+}$neurons (Fig. 6d). Collectively, these data indicate that impaired mitochondrial function might underlie the mechanism of $\left[\mathrm{Ca}^{2+}\right]_{\mathrm{cyt}}$ dyshomeostasis in $\mathrm{SOD} 2^{-1-}$ neurons.

\section{Intracellular $\mathrm{Ca}^{2+}$ chelation rescues mitochondrial and neurite outgrowth phenotypes in neurons lacking SOD2}

To determine whether disruption of $\mathrm{Ca}^{2+}$ homeostasis mediates the adverse effects of SOD2 deficiency on mitochondrial dynamics and neurite outgrowth in embryonic cortical neurons, we treated $\mathrm{SOD} 2^{+/+}$and $\mathrm{SOD} 2^{-/-}$neurons with BAPTA-AM or vehicle on culture day 1 and then quantified Drp1 phosphorylation, neurite length and mitochondrial functionality in neurons on culture day 3. Levels of p-Drp1 (Ser637), but not total Drp1, were significantly increased in BAPTA-AM treated $\mathrm{SOD} 2^{+/+}$and neurons (Fig. 7a). In SOD2 $2^{-/-}$neurons, BAPTA-AM treatment significantly increased levels of both p-Drp1 (Ser637) and total Drp1 to levels similar to those of control SOD2 $2^{+/+}$ neurons (Fig. 7a). In a parallel experiment we treated neurons of each genotype with BAPTA-AM or vehicle (culture days 1-3) and then quantified mitochondrial density in neurites and neurite length. Intracellular $\mathrm{Ca}^{2+}$ chelation did not affect the numbers of mitochondria in neurites nor the neurite length in SOD2 $2^{+/+}$and SOD2 $2^{+/-}$neurons (Fig. 7bd). However, in SOD2 $2^{-/-}$neurons, BAPTA-AM treatment significantly increased numbers of mitochondria in neurites and neurite length (Fig. 7b-d). To determine the contributions of reduced ATP levels, mitochondrial superoxide and $\mathrm{Ca}^{2+}$ to mitochondrial impairment in $\mathrm{SOD} 2^{-1-}$ neurons we treated SOD2 $2^{-1-}$ neurons with vehicle, the energy substrates uridine and pyruvate, the mitochondrial superoxide scavenger MitoTEMPO, BAPTA-AM or cyclosporin A (an inhibitor of membrane permeability transition pore formation) from culture days $1-3$. We then measured levels of cellular MTT reduction, an indicator of mitochondrial function. SOD2 $2^{-/-}$neurons treated with MitoTEMPO or BAPTA-AM exhibited significantly greater MTT reduction levels than did vehicle-treated neurons, whereas uridine/pyruvate and cyclosporin A had no significant effect on MTT reduction levels (Fig. 7f).

\section{Discussion}

Mitochondrial superoxide production negatively regulates neural progenitor cell proliferation and that SOD2 deficiency results in a reduction of progenitor cell self-renewal and thinning of the cerebral wall in mid-gestational embryonic mouse frontal cortex [33]. Mitochondria in the dendrites and axons serve as a critical local source of the ATP necessary for the function of ion-motive ATPases and other enzymes critical for electrochemical homeostasis, neurite outgrowth and neuroplasticity [1, 34]. In addition, mitochondria play important roles in the local regulation of subcellular $\mathrm{Ca}^{2+}$ dynamics in dendrites and axons [3, 35]. Vigorous mitochondrial biogenesis occurs as developing neurons rapidly grow neurites, the mitochondria are distributed throughout the length of the neurites [2, 36], and the movement of mitochondria along the length of neurites is regulated by $\mathrm{Ca}^{2+}$ [37]. Our findings suggest that mitochondrial SOD2 is necessary for proper regulation of cellular $\mathrm{Ca}^{2+}$ homeostasis which, in turn, enables mitochondrial dynamics and neurite outgrowth. 
A
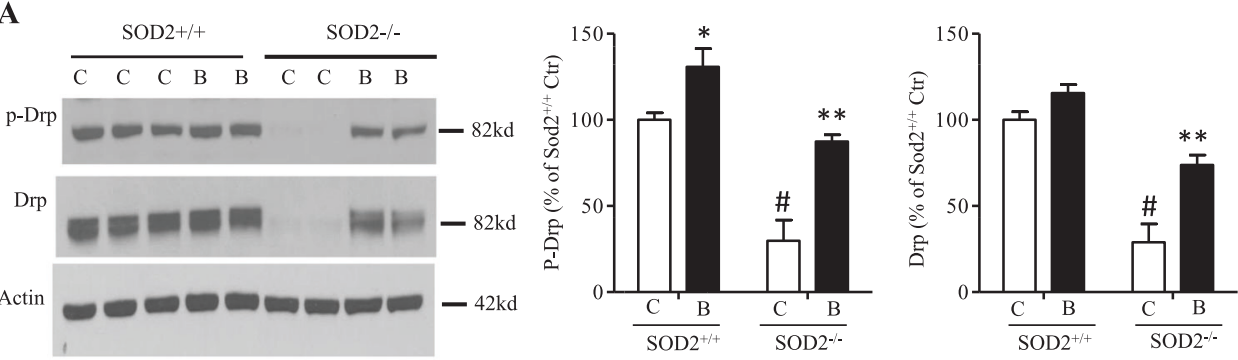

B
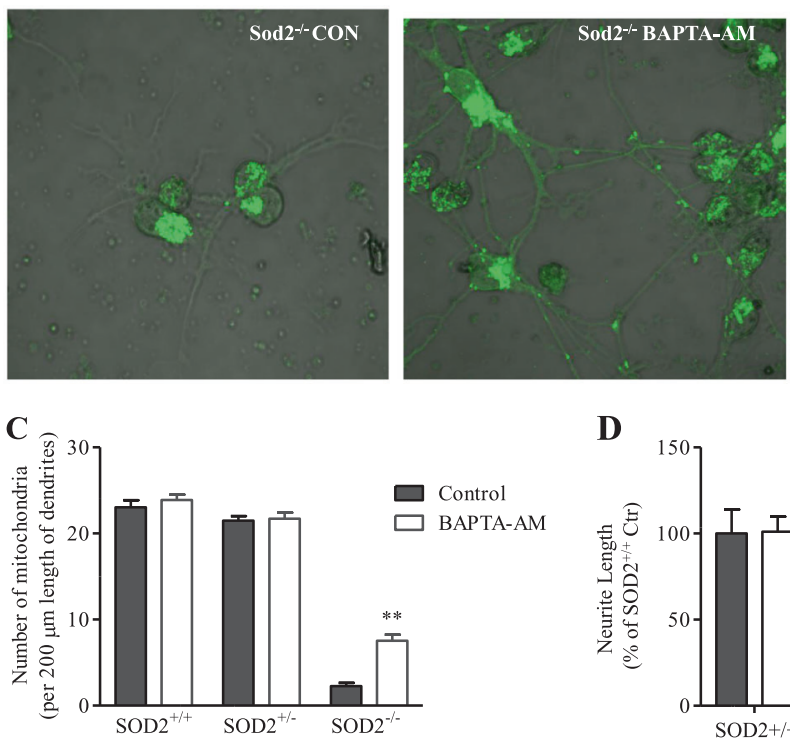

D

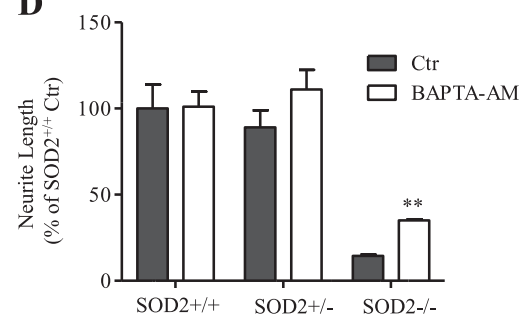

$\mathbf{E}$

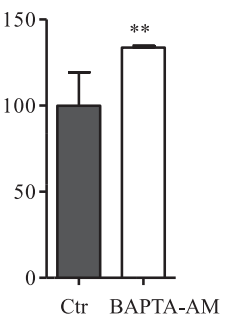

F

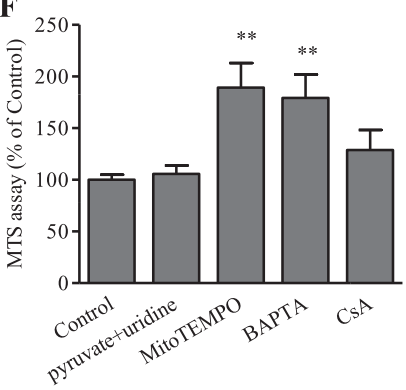

While mitochondrial fission was increased, and mitochondria were not properly distributed into the neurites of differentiating cortical neurons lacking SOD2, our data suggest that there was no significant effect of SOD2 deficiency on total mitochondrial mass. Thus, levels of the mitochondrial voltage-dependent anion-selective channels (VDAC) are not altered in SOD $2^{-/-}$neurons compared with that of SOD2 ${ }^{+/-}$or $\mathrm{SOD}^{+/+}$neurons during the first 3 days in culture (Fig. 2b). There was also no significant effect of SOD2 deficiency on the fluorescence intensity of Mitotracker Green per neuron (data not shown). We did find that cellular ATP levels were reduced in $\mathrm{SOD}^{-1-}$ neurons suggesting impaired mitochondrial function. Moreover, compared to control wild type neurons, neurons lacking
SOD2 or wild type neurons treated with the mitochondrial ATPase inhibitor oligomycin exhibited an impaired recovery of $\left[\mathrm{Ca}^{2+}\right]_{\mathrm{cyt}}$ when exposed to the mitochondrial uncoupling agent FCCP, indicating a key role for SOD2 in neuronal $\mathrm{Ca}^{2+}$ regulation. Thus impaired mitochondrial $\mathrm{Ca}^{2+}$ handling was associated with a significant basal $\left[\mathrm{Ca}^{2+}\right]_{\text {cyt }}$ elevation in SOD2 ${ }^{-/-}$neurons which occurred as early as two days in cultures. Furthermore, we found that the phosphorylation of Drp-1 on Ser 637 was strongly influenced by the $\left[\mathrm{Ca}^{2+}\right]_{\text {cyt }}$, being decreased in response to glutamate, and in SOD2 deficient neurons with elevated $\left[\mathrm{Ca}^{2+}\right]_{\mathrm{cyt}}$. The abnormal suppression pDrp1 (Ser 637) in $\mathrm{SOD} 2^{-l-}$ neurons would break the equilibrium of mitochondrial fission and fusion, the dynamic properties which 
Fig. 7 The $\mathrm{Ca}^{2+}$ chelator BAPTA-AM restores Drp1 phosphorylation and enhances neurite outgrowth and cell survival in SOD2 ${ }^{-/-}$cortical neurons. a Immunoblot analysis of p-Drp1, (S637) and Drp1 in $\mathrm{SOD}^{+/+}$and $\mathrm{SOD}^{-1-}$ cortical neurons (3 days in culture) that had been incubated with or without BAPTA-AM beginning on the day of cell plating. C: Control; B: BAPTA-AM $(1 \mu \mathrm{M})$. The blots were reprobed with antibodies against actin. The right panels are the results of densitometric analysis of blots showing p-Drp1 protein levels (percentage change compared to the value for SOD2 $2^{+/+}$neurons). Values are mean $\pm \mathrm{SD}$ ( $n=4$ cultures from separate embryos). $* * * p<0.001$ (ANOVA with student Newman-Keuls post-hoc tests). b Confocal image showing the merged images of phase-contrast and Mitotracker green fluorescence in SOD2 $2^{-/}$neurons (3 days in culture) that had been incubated with or without BAPTA-AM beginning on the day of cell plating. c, d Results of measurements of mitochondrial numbers per $200 \mu \mathrm{m}$ (c) and total neurite length (d) in SOD $2^{+/+}, \mathrm{SOD}^{+/-}$, and SOD $2^{-l-}$ neurons treated with or without BAPTA-AM. Values are mean \pm SEM $\left(n=3-4\right.$ separate cultures). ${ }^{*} p<0.01$. (ANOVA with student Newman-Keuls post-hoc tests). e ATP levels in primary cultured SOD $2^{-1-}$ cortical neurons (3 days in culture) treated with or without BAPTA-AM. The data are presented as percentage of the cultures without BAPTA-AM treatment $(\mathrm{Ctr}) . * * p<0.01$ compared to the $\mathrm{Ctr}$ value. Values are mean $\pm \mathrm{SD}$ ( $n=$ cultures from 3 embryos). $\mathbf{f}$ Results of quantitative analysis of neuronal survival in cultures of $S O D 2^{-/-}$cortical neurons that had been cultured in presence of pyruvate $(100 \mu \mathrm{g} / \mathrm{ml})$ and uridine $(50 \mu \mathrm{g} / \mathrm{ml})$, mitoTEMPO $(1 \mu \mathrm{M}$; a mitochondrial superoxide scavenger), MnTMPyP $(1 \mu \mathrm{M}$; a cell permeable SOD mimetic), cyclosporin A (CsA, $500 \mathrm{nM}$; a mitochondrial PTP inhibitor) or FK506 (10 nM; a calcineurin inhibitor). Cell survival was quantified by MTS assays. Values are mean \pm SEM (separate cultures from 4 mice). $* * p<0.05$ and $* * * P<0.01$

are important for functional output of mitochondria as the powerhouse of the cells [38]. Finally, we found that treatment of embryonic cortical neurons with the $\mathrm{Ca}^{2+}$ chelator BAPTA-AM prevented Drp-1 dephosphorylation in SOD2 ${ }^{-/-}$neurons and in glutamate treated $\mathrm{SOD} 2^{+/+}$neurons. BAPTA-AM treatment also prevented mitochondrial fission, and enabled neurite outgrowth and distribution of mitochondria into the growing neurites in $\mathrm{SOD} 2^{-/-}$neurons. These findings suggest that SOD2 is critical for the regulation of $\left[\mathrm{Ca}^{2+}\right]_{\mathrm{cyt}}$, which, in turn, is necessary for the maintenance of a normal balance of mitochondrial fission and fusion and for the normal distribution of mitochondria in growing neurites (Fig. 8).

In contrast to SOD2 knockout mice which die during late gestation or soon after birth [15, 24], SOD1 and SOD3 knockout mice survive into adulthood and old age [13, 39]. Only a few and relatively subtle abnormalities in the nervous system of SOD1 deficient mice have been described including a moderate decrease in motor unit size and a distal motor neuron axonopathy, but with no change in the number and size of motor neurons [40, 41]. However, neurons in the cerebral cortex and striatum of SOD1 deficient mice exhibit increased vulnerability to ischemic injury and death in an experimental stroke model [42]. SOD3 knockout mice have a normal lifespan [13] and no neurological phenotypes have been described in the SOD3 deficient mice. Cytoplasmic and plasma membraneassociated oxidases such as NADPH oxidase can also generate superoxide [43], but NADPH deficient mice appear to develop normally and live into adulthood [44] with no apparent abnormalities in brain development [45]. The present findings suggest that SOD2 plays critical roles in maintenance of mitochondrial and cytosolic $\mathrm{Ca}^{2+}$ homeostasis. Moreover, by maintaining Drp1 phosphorylation, as well as Drp1 levels, SOD2 plays important roles in regulating mitochondrial fission and fusion dynamics, and thus mitochondria integrity, distribution and functions (Fig. 8).

\section{Materials and methods}

\section{SOD2 deficient mice and primary neuronal cultures}

Breeding pairs of $S O D 2^{+/-}$mice $(\operatorname{Sod} 2 \mathrm{tm} 1 \mathrm{Leb})$ were purchased from Jackson Laboratories (Bar Harbor, ME). The colony was maintained, and littermate experimental animals were generated by breeding $S O D 2^{+/-}$males with $S O D 2^{+/-}$females. For genotyping, two complementary polymerase chain reactions were performed on genomic DNA from adult mouse tails or embryonic mouse tissues. The first reaction used primers for detecting a SOD2 exon 1 sequence (5'-ACG TTG CCT TCC CAG GAT-3' and 5'GTT TAC ACG ACC GCT GCT CT- $3^{\prime}$ ), and the second reaction used primers for detecting a mutated sequence $\left(5^{\prime}\right.$ TGT TCT CCTCTT CCT CAT CTC C- $3^{\prime}$ and $5^{\prime}$-ACC CTT TCC AAA TCCTCA GC-3'). The first reaction generates a 193-bp product for the wild type (WT) allele, and the second reaction generates a 240 -bp product for the mutant allele. For primary cultures of embryonic cortical neurons, timed-pregnant mice were obtained by breeding $S O D 2^{+/-}$male and female mice. Cultures of embryonic cortical neurons were prepared from embryonic day (E) 14.5 cerebral tissues as described previously $[2,17]$. The dissociated cells were seeded into polyethyleneiminecoated plastic culture dishes or glass coverslips at a density of 50,000 cells $/ \mathrm{cm}^{2}$. After a 4-h incubation in MEM containing $10 \%$ fetal bovine serum, the medium was replaced with Neurobasal medium containing B27 supplements, $2 \mathrm{mM}$ L-glutamine, antibiotic antimycotic (Gibco) and $1 \mathrm{mM}$ HEPES (all from Invitrogen, Carlsbad, CA). The culture treatments included: L-glutamic acid (Glu), BAPTA-AM, FK506, okadaic acid (OA), cyclosporin A (CSA), MnTMPyp, MitoTEMPO, pyruvate, uridine, oligomycin (Oligo), carbonyl cyanide 4(trifluoromethoxy) phenylhydrazone (FCCP). All these reagents were from Sigma, St. Louis, MO and were prepared as $500-1000 \times$ stocks in dimethylsulfoxide or distilled water. Treatments were administered by direct 


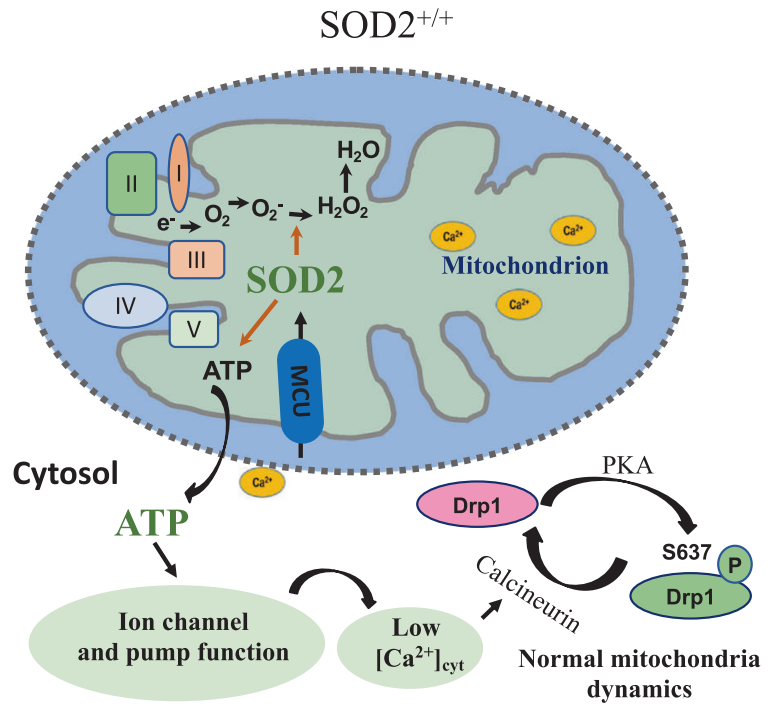

Normal mitochondrial morphology Normal mitochondrial distribution Neurite outgrowth

Fig. 8 Working model for the role of SOD2 in enabling neurite outgrowth and neuronal survival by mechanisms involving maintenance of mitochondrial and cytosolic $\mathrm{Ca}^{2+}$ homeostasis and Drp1 phosphorylation. a SOD2 sustains mitochondrial function and integrity by preventing dysregulation of mitochondrial $\mathrm{Ca}^{2+}$ handling and electron transport. As a result, ATP levels are maintained, cytosolic $\mathrm{Ca}^{2+}$ levels are maintained within normal limits, Drp1 remains in a phosphorylated state, and mitochondria maintain a normal balance of fission and fusion. By enabling mitochondria to perform their ATP-generating and

dilution into the culture medium, and an equivalent volume of vehicle was added to control cultures.

\section{Immunoblot analysis}

Tissues or cultured cells were solubilized in SDSpolyacrylamide gel electrophoresis sample buffer, and the protein concentration in each sample was determined using a Bio-Rad protein assay kit with bovine serum albumin as the standard. Proteins ( $30 \mu \mathrm{g}$ of protein per lane) were then separated in 4-12\% SDS gels (Invitrogen, Carlsbad, CA) using electrophoresis, and then electrophoretically transferred to a nitrocellulose membrane. Membranes were blocked with $4 \%$ nonfat milk in TBST (Tris- $\mathrm{HCl}$ based buffer with $0.2 \%$ Tween $20, \mathrm{pH} 7.5$ ), and then incubated for $2 \mathrm{~h}$ in the presence of primary antibody. Cells were then incubated for $1 \mathrm{~h}$ in the presence of a 1:5000 dilution of secondary antibody ( $\mathrm{IgG}$ ) conjugated to horseradish peroxidase. Reaction product was visualized using an Enhanced Chemiluminescence (ECL) Western blot detection kit (Amersham Pharmacia Biotech, United Kingdom). The primary antibodies included those specific for SOD2 (rabbit, 1:2000, Enzo Life Sciences, Farmingdale, NY), Drp and Drp (S637 phosphorylation) (rabbit, 1:500, Cell signaling technology, Danvers, MA), VDAC (rabbit, 1:1000,

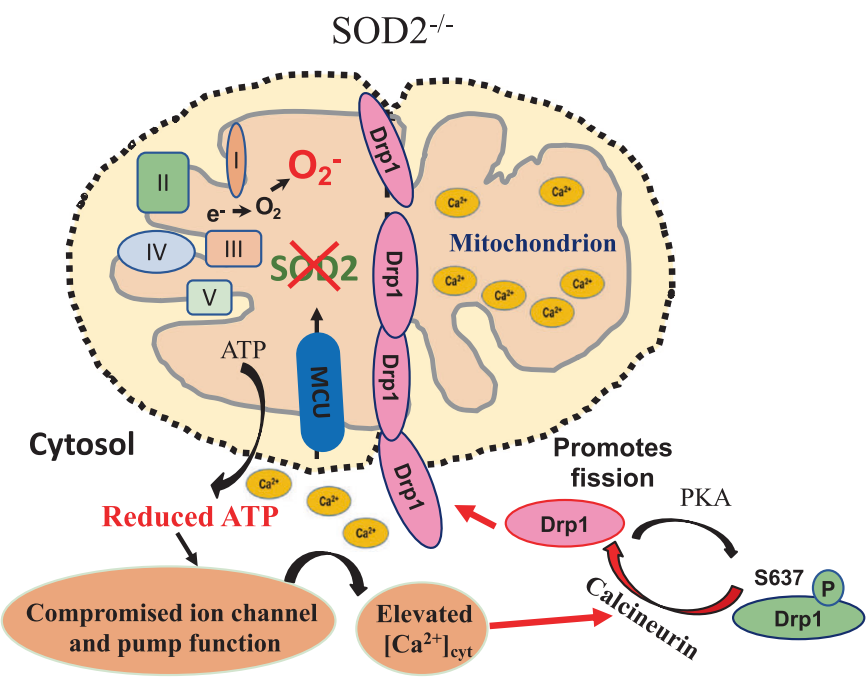

\begin{abstract}
Abnormal mitochondrial fragmentation
Disturbed mitochondrial distribution

Neurite outgrowth suppression

Neuronal death
\end{abstract}

$\mathrm{Ca}^{2+}$-regulating functions, SOD2 plays a critical role in supporting neurite outgrowth in embryonic cerebral cortical neurons. b When SOD2 expression or activity is reduced, mitochondrial ATP production and $\mathrm{Ca}^{2+}$ homeostasis are compromised resulting in elevation of cytoplasmic $\mathrm{Ca}^{2+}$ levels, calcineurin activation and dephosphorylation of Drp1. Drp1 then translocates to mitochondria where it triggers abnormal mitochondrial fission which is associated with accumulation of dysfunctional mitochondria in the neuronal cell body and impaired neurite outgrowth

Abcam, Cambridge, MA), actin (mouse, Sigma, St. Louis, MO, 1:2000).

\section{Neuronal viability assays}

Hoechst staining and MTT assays were performed to evaluate neuronal death or viability. For Hoechst staining, primary cultured neurons were stained with the DNA-binding dye Hoechst 33342 by direct addition to the culture medium for $30 \mathrm{~min}$ to $1 \mathrm{~h}$. Cells were observed and images of 5 randomly selected fields were acquired using a digital camera connected to Zeiss fluorescence microscope. Phase-contrast and Hoechst fluorescence images were simultaneously acquired using a $\times 20$ objective under the fluorescence microscope with the white light and UV light channels both on. The number of dead and viable neurons were quantified using NIH Image J software. Cells were considered dead if their nuclear chromatin stained nuclei are bright, condensed or fragmented, whereas cells were considered viable if their chromatin was diffusely and evenly distributed throughout the nucleus with intact neuronal morphology. For MTT assay, neurons in 96-well plates seeded at the same density under control or treatment conditions were exposed to MTS (Promega, Madison, 
WI, USA) for $3-4 \mathrm{~h}$ at $37^{\circ} \mathrm{C}$. The absorbance of the MTS formazan products was measured at $490 \mathrm{~nm}$. MTT is a pale-yellow substrate that is cleaved by living cells to yield a dark blue formazan product. This process requires functional mitochondrial activity [46, 47].

\section{Immunocytochemistry, mitochondria labeling and confocal imaging}

For immunofluorescence cytochemistry, fixed cells were preincubated with blocking solution $(0.2 \%$ Triton $\mathrm{X}-100$, $10 \%$ normal goat serum) in PBS for $30 \mathrm{~min}$, and then incubated overnight with a primary antibody followed with appropriate secondary antibodies for $2 \mathrm{~h}$ at room temperature. The cells were counterstained with DAPI for $10 \mathrm{~min}$. The primary antibody was rabbit beta-3 tubulin (TuJ1) (1: 200 dilution; Sigma), rabbit Drp1 (1:100, Cell Signaling Technology) and mouse OxPhos-complex IV subunit 1 (COX1, 1:100; Invitrogen). The secondary antibody was FITC or Rhodamine- conjugated goat anti-rabbit or mouse (1:500, Molecular Probes). To label mitochondria, the cultured neurons were loaded with Mitotracker Green (200 $\mathrm{nM}, 30 \mathrm{~min}$ incubation). Images were acquired using the confocal with an Olympus Multiphoton Laser Scanning Microscope with $\times 60(\mathrm{NA}, 0.9)$ objectives. Total neurite length per neuron and mitochondria number and length were quantified using Olympus Fluoview (FV10-ASW2.1) software and analyzed and plotted by micro-soft Excel and GraphPad Prism 5.

\section{Adeno-associated virus (AAV) construction and packaging}

pcDNA3.1-mito-GCaMP3 was constructed by Heping Cheng's lab (Institute of Molecular Medicine, Peking University, China). The PCR products of mito-GCaMP3 cDNA were ligated with PCR Blunt II TOPO vector and subcloned into pAAV MCS vector by EcoRI. For packaging, AAVGFP or AAV-mito-GCAMP3 were co-transfected with pAAV-DJ vector and pHelper vector (Cell Biolab) into HEK293 cells (newly seeded and grown to approximately $60 \%$ confluency) at the molar ratio of 1:1:1 using a CalPhos $^{\mathrm{TM}}$ Mammalian Transfection Kit (Clontech). At 48-72 $\mathrm{h}$ after transfection, cells were collected and pelleted by centrifugation and high titer stocks of recombinant AAV viruses were purified using iodixanol gradient solutions and ultra-centrifuged at $36,6585 \mathrm{Rcf}(\mathrm{g})$ for $1 \mathrm{~h}$. The virus collected from the iodixanol gradient was concentrated and purified in sterile PBS using centrifugal filter units (Amicon Ultra). The concentrated virus was transduced into primary cultured neurons at a series of dilutions to observe GFP expression under fluorescence microscopy to determine the desired dilutions.

\section{Calcium measurements}

Primary cultured neurons were incubated for $30 \mathrm{~min}$ at 37 ${ }^{0} \mathrm{C}$ with Fluo-3-AM $(1 \mu \mathrm{M})$ and Fura-Red-AM $(5 \mu \mathrm{M})$ in Locke's solution $(154 \mathrm{mM} \mathrm{NaCl}, 5.6 \mathrm{mM} \mathrm{KCl}, 2.3 \mathrm{mM}$ $\mathrm{CaCl}_{2}, 1.0 \mathrm{mM} \mathrm{MgCl}, 3.6 \mathrm{mM} \mathrm{NaHCO} 3,20 \mathrm{mM}$ Glucose, $20 \mathrm{mM}$ HEPES). Calcium dyes were obtained from Thermo Fisher and dissolved in dimethylsulfoxide at a stock concentration of $1 \mathrm{mM}$. Scanning confocal imaging was achieved using an Olympus Multiphoton Laser Scanning Microscope with a LUMPLEL $\times 60,0.9$ N.A. water immersion objective lens. The $488 \mathrm{~nm}$ line of an argon laser was used for excitation. The fluorescence light was longpass filtered and separated by a beam filter into two wavelength bands: from 505-605 nm (Fluo-3 channel, wavelength 1) and from $605-755 \mathrm{~nm}$ (Fura Red channel, wavelength 2) respectively, before detection with two photomultipliers. Frames of $512 \times 512$ pixel or $1024 \times 1024$ pixels were taken at a sampling rate of $2 \mathrm{~s} /$ frame. The total number of frames and the length of imaging were dependent upon the experimental design. Imaging experiments were performed at room temperature $\left(24-26^{\circ} \mathrm{C}\right)$. Digital image processing used Olympus Fluoview (FV10-ASW2.1) software and Microsoft Office Excel and user-designed programs.

The calcium indicators Fluo-3 and Fura-red were used as a ratiometric pair to measure the cytosolic calcium concentration $\left(\left[\mathrm{Ca}^{2+}\right]_{\mathrm{cyt}}\right)$. When the ratio of fluo-3/Fura-red were determined, the $\left[\mathrm{Ca}^{2+}\right]_{\text {cyt }}$ was calculated using the following equation, which describes the correlation between $\left[\mathrm{Ca}^{2+}\right]_{\mathrm{cyt}}$ and the fluorescent intensity ratio of Fluo-3/Furared:

$R=\frac{F 1}{F 2}=\frac{\frac{C F_{\max , 1}}{K_{d l}+C}+\frac{K_{d l} F_{\text {min }, 1}}{K_{d d}+C}}{\frac{C F_{\min , 2}}{K_{d 2}+C}+\frac{K_{d 2} F_{\max , 2}}{K_{d 2}+C}}$

In this equation, $C$ represents $\left[\mathrm{Ca}^{2+}\right]_{\text {cyt }} ; R$ is fluorescence intensity ratio of Fluo-3/Fura-red collected at corresponding channels as described above; $K_{\mathrm{d} 1}$ and $K_{\mathrm{d} 2}$ are dissociation constant for Fluo-3 and Fura-red, respectively in cells; $F_{\text {max }, 1}$ and $F_{\text {min, } 1}$ are the $\mathrm{Ca}^{2+}$-saturated and $\mathrm{Ca}^{2+}$-free Fluo3 fluorescence intensities, respectively, at wavelength 1; $F_{\max , 2}$, and $F_{\min , 2}$ are the free and full bound Fura-red, respectively, at wavelength $2 \cdot \frac{C}{K_{d}+C}(=f b)$, is the fraction of the indicator in the $\mathrm{Ca}^{2+}$-bound form and $\frac{K_{d}}{K_{d}+C}(=f f)$, is the fraction of the indicator in the $\mathrm{Ca}^{2+}$ free form.

To determine $K_{\mathrm{d}}$ of Fluo-3 and Fura-Red as a ratiometric pair of $\left[\mathrm{Ca}^{2+}\right]_{\text {in }}$ indicators in living neurons, the calcium calibration buffers with a series of concentration $(0,0.017$, $0.038,0.065,0.100,0.150,0.225,0.351,0.602,1.35$, and $39 \mu \mathrm{M}$ ) of free calcium were prepared as the standard protocol of the calcium calibration buffer Kits (Molecular Probes, C3008 MP). The calcium ionophore ionomycin 
$(10 \mu \mathrm{M})$ was included in the calibration solutions to enable equilibration of $\mathrm{Ca}^{2+}$ across the plasma membrane. Cells loaded with Fluo-3 $(1 \mu \mathrm{M})$ and Fura Red $(5 \mu \mathrm{M})$ were incubated $10 \mathrm{~min}$ in the calibration solutions before images were acquired. The fluorescence intensity ratio of Fluo-3/ Fura-red was plotted against the $\left[\mathrm{Ca}^{2+}\right]_{\text {cyt }}$ and fitted by nonlinear least squares method. The optimal best-fit pair of $K_{\mathrm{d} 1}$ (for Fluo-3) and $K_{\mathrm{d} 2}$ (for Fura-red) for $\left[\mathrm{Ca}^{2+}\right]_{\text {cyt }}$ calculation, and the corresponding $F_{\max , 1}$ and $F_{\min , 1}, F_{\max , 2}$, and $F_{\min , 2}$ were simultaneously obtained. Under the conditions of our study the $K_{\mathrm{d} 1}$ for Fluo-3 was $1396.8 \mathrm{nM}, K_{\mathrm{d} 2}$ for Fura-red was $343 \mathrm{nM}, F_{\min , 1}$ was 1005.5 and $F_{\max , 1}$ was 4095 (fluo-3), $F_{\min , 2}$ was 828.1 and $F_{\max , 2}$ was 3372.5 (Fura-red).

\section{Statistical analyses}

Statistical analyses were performed by one-way ANOVA or $t$-test using GraphPad Prism (GraphPad, San Diego, CA, USA). Newman-Keuls post hoc tests were applied to detect statistical differences between groups.

Acknowledgements This research was supported by the Intramural Research Program of the National Institute on Aging and NSFC (Project 31521062).

\section{Compliance with ethical standards}

Conflict of interest The authors declare that they have no conflict of interest.

\section{References}

1. Mattson MP, Gleichmann M, Cheng A. Mitochondria in neuroplasticity and neurological disorders. Neuron. 2008;60: 748-66.

2. Cheng A, Wan R, Yang J, Kamimura N, Son TG, Ouyang X, et al. Involvement of PGC- $1 \alpha$ in the formation and maintenance of neuronal dendritic spines. Nat Commun. 2012;3:1250.

3. Li Z, Okamoto K, Hayashi Y, Sheng M. The importance of dendritic mitochondria in the morphogenesis and plasticity of spines and synapses. Cell. 2004;119:873-87.

4. Vayssière JL, Cordeau-Lossouarn L, Larcher JC, Basseville M, Gros F, Croizat B. Participation of the mitochondrial genome in the differentiation of neuroblastoma cells. Vitr Cell Dev Biol. 1992;28A:763-72.

5. Mattson MP, Partin J. Evidence for mitochondrial control of neuronal polarity. J Neurosci Res. 1999;56:8-20.

6. Khacho M, Slack RS. Mitochondrial dynamics in the regulation of neurogenesis: from development to the adult brain. Dev Dyn. 2018;247:47-53

7. Knapp LT, Klann E. Role of reactive oxygen species in hippocampal long-term potentiation: contributory or inhibitory? J Neurosci Res. 2002;70:1-7.

8. Andersen JK. Oxidative stress in neurodegeneration: cause or consequence? Nat Med. 2004;10:Suppl: S18-25.

9. Nicholls DG, Vesce S, Kirk L, Chalmers S. Interactions between mitochondrial bioenergetics and cytoplasmic calcium in cultured cerebellar granule cells. Cell Calcium. 2003;34:407-24.
10. Giacomello M, Drago I, Pizzo P, Pozzan T. Mitochondrial $\mathrm{Ca}^{2+}$ as a key regulator of cell life and death. Cell Death Differ. 2007;14:1267-74.

11. Yang JL, Tadokoro T, Keijzers G, Mattson MP, Bohr VA. Neurons efficiently repair glutamate-induced oxidative DNA damage by a process involving CREB-mediated up-regulation of apurinic endonuclease 1. J Biol Chem. 2010;285:28191-9.

12. Carlsson LM, Jonsson J, Edlund T, Marklund SL. Mice lacking extracellular superoxide dismutase are more sensitive to hyperoxia. Proc Natl Acad Sci USA. 1995;92:6264-8.

13. Sentman ML, Granström M, Jakobson H, Reaume A, Basu S, Marklund SL. Phenotypes of mice lacking extracellular superoxide dismutase and copper- and zinc-containing superoxide dismutase. J Biol Chem. 2006;281:6904-9.

14. Li Y, Huang TT, Carlson EJ, Melov S, Ursell PC, Olson JL, et al. Dilated cardiomyopathy and neonatal lethality in mutant mice lacking manganese superoxide dismutase. Nat Genet. 1995;11:376-81.

15. Lebovitz RM, Zhang H, Vogel H, Cartwright J Jr, Dionne L, Lu $\mathrm{N}$, et al. Neurodegeneration, myocardial injury, and perinatal death in mitochondrial superoxide dismutase-deficient mice. Proc Natl Acad Sci USA. 1996;93:9782-7.

16. Andreassen OA, Ferrante RJ, Dedeoglu A, Albers DW, Klivenyi $\mathrm{P}$, Carlson EJ, et al. Mice with a partial deficiency of manganese superoxide dismutase show increased vulnerability to the mitochondrial toxins malonate, 3-nitropropionic acid, and MPTP. Exp Neurol. 2001;167:189-95.

17. Cheng A, Yang Y, Zhou Y, Maharana C, Lu D, Peng W, et al. Mitochondrial SIRT3 mediates adaptive responses of neurons toexercise and metabolic and excitatory challenges. Cell Metab. 2016;23:128-42.

18. Keller JN, Kindy MS, Holtsberg FW, St Clair DK, Yen HC, Germeyer A, et al. Mitochondrial manganese superoxide dismutase prevents neural apoptosis and reduces ischemic brain injury: suppression of peroxynitrite production, lipid peroxidation, and mitochondrial dysfunction. J Neurosci. 1998;18:687-97.

19. Massaad CA, Washington TM, Pautler RG, Klann E. Overexpression of SOD-2 reduces hippocampal superoxide and prevents memory deficits in a mouse model of Alzheimer's disease. Proc Natl Acad Sci USA. 2009;106:13576-81.

20. Mattson MP, Dou P, Kater SB. Outgrowth-regulating actions of glutamate in isolated hippocampal pyramidal neurons. J Neurosci. 1988;8:2087-100.

21. Mattson MP, Murain M, Guthrie PB. Localized calcium influx orients axon formation in embryonic hippocampal pyramidal neurons. Brain Res Dev Brain Res. 1990;52:201-9.

22. Mattson MP, Lee RE, Adams ME, Guthrie PB, Kater SB. Interactions between entorhinal axons and target hippocampalneurons: a role for glutamate in the development of hippocampalcircuitry. Neuron. 1988;1:865-76.

23. Flippo KH, Strack S. Mitochondrial dynamics in neuronal injury, development and plasticity. J Cell Sci. 2017;130:671-81.

24. Friedman JS, Rebel VI, Derby R, Bell K, Huang TT, Kuypers FA, et al. Absence of mitochondrial superoxide dismutase results in a murine hemolytic anemia responsive to therapy with a catalytic antioxidant. J Exp Med. 2001;193:925-34.

25. Knott AB, Perkins G, Schwarzenbacher R, Bossy-Wetzel E. Mitochondrial fragmentation in neurodegeneration. Nat Rev Neurosci. 2008;9:505-18.

26. Cereghetti GM, Stangherlin A, Martins de Brito O, Chang CR, Blackstone C, Bernardi P, et al. Dephosphorylation by calcineurin regulates translocation of Drp1 to mitochondria. Proc Natl Acad Sci USA. 2008;105:15803-8.

27. Chang CR, Blackstone C. Cyclic AMP-dependent protein kinase phosphorylation of Drp1 regulates its GTPase activity and mitochondrial morphology. J Biol Chem. 2007;282:21583-7. 
28. Floto RA, Mahaut-Smith MP, Somasundaram B, Allen JM. (1995) IgG-induced Ca2+oscillations in differentiated U937 cells; a study using laser scanning confocal microscopy and co-loaded fluo-3 and fura-red fluorescent probes. Cell Calcium. 1995;18:377-89.

29. Schild D, Jung A, Schultens HA. Localization of calcium entry through calcium channels in olfactory receptor neurons using a laser scanning microscope and the calcium indicator dyes Fluo-3 and Fura-Red. Cell Calcium. 1994;15:341-8.

30. Lipp P, Niggli E. Ratiometric confocal $\mathrm{Ca}(2+)$-measurements with visible wavelength indicators in isolated cardiac myocytes. Cell Calcium. 1993;14:359-72.

31. Harkins AB, Kurebayashi N, Baylor SM. Resting myoplasmic free calcium in frog skeletal muscle fibers estimated with fluo-3. Biophys J. 1993;65:865-81.

32. Kurebayashi N, Harkins AB, Baylor SM. Use of fura red as an intracellular calcium indicator in frog skeletal muscle fibers. Biophys J. 1993;64:1934-60.

33. Hou Y, Ouyang X, Wan R, Cheng H, Mattson MP, Cheng A. Mitochondrial superoxide production negatively regulates neural progenitor proliferation and cerebral cortical development. Stem Cells. 2012;30:2535-47.

34. Fukumitsu K, Fujishima K, Yoshimura A, Wu YK, Heuser J, Kengaku M. Synergistic action of dendritic mitochondria and creatine kinase maintains ATP homeostasis and actin dynamics in growing neuronal dendrites. J Neurosci. 2015;35:5707-23.

35. Mattson MP. Mitochondrial regulation of neuronal plasticity. Neurochem Res. 2007;32:707-15.

36. Ligon LA, Steward O. Movement of mitochondria in the axons and dendrites of cultured hippocampal neurons. J Comp Neurol. 2000;427:340-50.

37. Niescier RF, Chang KT, Min KT. Miro, MCU, and calcium: bridging our understanding of mitochondrial movement in axons. Front Cell Neurosci. 2013;7:148.

38. Chan DC. Mitochondria: dynamic organelles in disease, aging, and development. Cell. 2006;125:1241-52.
39. Elchuri S, Oberley TD, Qi W, Eisenstein RS, Jackson Roberts L, Van Remmen H, et al. CuZnSOD deficiency leads to persistent and widespread oxidative damage and hepatocarcinogenesis later in life. Oncogene. 2005;24:367-80.

40. Shefner JM, Reaume AG, Flood DG, Scott RW, Kowall NW, Ferrante RJ, et al. Mice lacking cytosolic copper/zinc superoxide dismutase display a distinctive motor axonopathy. Neurology. 1999;53:1239-46.

41. Fischer LR, Li Y, Asress SA, Jones DP, Glass JD. Absence of SOD1 leads to oxidative stress in peripheral nerve and causes a progressive distal motor axonopathy. Exp Neurol. 2012;233:163-71.

42. Kondo T, Reaume AG, Huang TT, Murakami K, Carlson E, Chen $\mathrm{S}$, et al. Edema formation exacerbates neurological and histological outcomes after focal cerebral ischemia in CuZn-superoxide dismutase gene knockoutmutant mice. Acta Neurochir Suppl. 1997;70:62-64.

43. Abramov AY, Canevari L, Duchen MR. Beta-amyloid peptides induce mitochondrial dysfunction and oxidative stress in astrocytes and death of neurons through activation of NADPH oxidase. J Neurosci. 2004;24:565-75.

44. Pollock JD, Williams DA, Gifford MA, Li LL, Du X, Fisherman $\mathrm{J}$, et al. Mouse model of X-linked chronic granulomatous disease, an inherited defect in phagocyte superoxide production. Nat Genet. 1995;9:202-9.

45. Kishida KT, Hoeffer CA, Hu D, Pao M, Holland SM, Klann E. Synaptic plasticity deficits and mild memory impairments in mouse models of chronic granulomatous disease. Mol Cell Biol. 2006;26:5908-20.

46. Berridge MV, Tan AS. Characterization of the cellular reduction of 3-(4,5-dimethylthiazol-2-yl)-2,5-diphenyltetrazolium bromide (MTT): subcellular localization, substrate dependence, and involvement of mitochondrial electron transport in MTT reduction. Arch Biochem Biophys. 1993;303:474-82.

47. Berridge M, Tan A, McCoy K, Wang R. The biochemical and cellular basis of cell proliferation assays that use tetrazolium salts. Biochemica. 1996;4:14-19. 